ARTICLE

DOI: $10.1038 / s 41467-018-04827-z$

\title{
Identification of dynamic undifferentiated cell states within the male germline
}

Hue M. La1,2, Juho-Antti Mäkelä (1) 1,2,3, Ai-Leen Chan,2, Fernando J. Rossello (1) 1,2, Christian M. Nefzger ${ }^{1,2}$, Julien M.D. Legrand (1) 1,2, Mia De Seram¹,2, Jose M. Polo (i) ${ }^{1,2} \&$ Robin M. Hobbs (10) ${ }^{1,2}$

The role of stem cells in tissue maintenance is appreciated and hierarchical models of stem cell self-renewal and differentiation often proposed. Stem cell activity in the male germline is restricted to undifferentiated $A$-type spermatogonia ( $A_{\text {undiff }}$ ); however, only a fraction of this population act as stem cells in undisturbed testis and $A_{\text {undiff }}$ hierarchy remains contentious. Through newly developed compound reporter mice, here we define molecular signatures of self-renewing and differentiation-primed adult $A_{\text {undiff }}$ fractions and dissect $A_{\text {undiff }}$ heterogeneity by single-cell analysis. We uncover an unappreciated population within the selfrenewing $A_{\text {undiff }}$ fraction marked by expression of embryonic patterning genes and homeodomain transcription factor PDX1. Importantly, we find that PDX1 marks a population with potent stem cell capacity unique to mature, homeostatic testis and demonstrate dynamic interconversion between PDX1+ and PDX1- $\mathrm{A}_{\text {undiff }}$ states upon transplant and culture. We conclude that $A_{\text {undiff }}$ exist in a series of dynamic cell states with distinct function and provide evidence that stability of such states is dictated by niche-derived cues.

\footnotetext{
${ }^{1}$ Australian Regenerative Medicine Institute, Monash University, Melbourne, VIC 3800, Australia. ${ }^{2}$ Development and Stem Cells Program, Monash Biomedicine Discovery Institute and Department of Anatomy and Developmental Biology, Monash University, Melbourne, VIC 3800 , Australia. ${ }^{3}$ Present address: Institute of Biomedicine, Research Centre for Integrative Physiology and Pharmacology, University of Turku, Turku, Finland. These authors contributed equally: Hue M. La, Juho-Antti Mäkelä. Correspondence and requests for materials should be addressed to R.M.H. (email: robin.hobbs@monash.edu)
} 
imited lifespan of differentiated cells in many tissues necessitates replacement to ensure tissue maintenance. Within such high turnover tissues, resident stem cells generate fresh cohorts of differentiating cells. Stem cell populations can be heterogeneous and composed of multiple pools with distinct functional characteristics and involvement in tissue maintenance and repair ${ }^{1}$. Stem cell activity can also be context dependent ${ }^{1}$. The ability to define different stem cell subsets within a tissue is dependent on availability of molecular markers that delineate populations.

Sustained spermatogenesis and recovery of fertility following germ cell depletion are dependent on stem cells within the testis seminiferous epithelium ${ }^{2}$. In adult mice, germline stem cell activity is contained within a population of undifferentiated Type A spermatogonia $\left(\mathrm{A}_{\text {undiff }}\right)$, which develop postnatally from foetal germ cells (gonocytes). Gonocytes migrate from the lumen of developing seminiferous tubules to the basement membrane and generate undifferentiated and differentiating spermatogonia. The adult $A_{\text {undiff }}$ pool contains isolated single cells (A-single or $A_{s}$ ) plus chains of cells remaining interconnected by cytoplasmic bridges after cell division; 2-cell chains are A-paired $\left(A_{p r}\right)$ while chains of 4 or more A-aligned $\left(A_{a l}\right)$. $A_{\text {undiff }}$ differentiation is marked by induction of c-KIT plus DNA methyltransferases $3 \mathrm{~A}$ and $3 \mathrm{~B}(\mathrm{DNMT} 3 \mathrm{~A} / 3 \mathrm{~B})$ and formation of $\mathrm{A}_{1}$ spermatogonia ${ }^{3} . \mathrm{A}_{1}$ cells undergo a series of mitotic divisions and via $A_{2}, A_{3}, A_{4}$, Intermediate (In) and $\mathrm{B}$ spermatogonia generate meiotic spermatocytes. It is generally accepted that germline stem cell activity is contained within the $A_{\text {undiff }}$ pool. However, $A_{\text {undiff }}$ cells are heterogeneous and contrasting models of $\mathrm{A}_{\text {undiff }}$ hierarchy and stem cell identity are proposed ${ }^{4,5}$.

A founding model of spermatogonial hierarchy proposed that $A_{s}$ act as stem cells while $A_{p r}$ and $A_{a b}$, the bulk of $A_{\text {undiff, }}$ are differentiation-committed progenitors ${ }^{2}$. Upon division, $A_{s}$ generate two $A_{s}$ for self-renewal or $A_{p r}$ that generate $A_{a l}$ and differentiate. This model has been challenged through transplantation and lineage tracing. Germline stem cells form long-lived spermatogenic colonies when injected into seminiferous tubules of germ cell-depleted recipients ${ }^{6}$. As each colony is normally derived from a single cell, stem cell numbers in the donor population can be estimated. While there are $\sim 35,000 \mathrm{~A}_{\mathrm{s}}$ in an adult testis, only $\sim 3000$ cells have transplant capability ${ }^{7}$, suggesting that not all $\mathrm{A}_{\mathrm{s}}$ are stem cells. Recent studies concluded that transplant activity is almost exclusively contained within a fraction of $\mathrm{A}_{\mathrm{s}}$ marked by $I d 4$ expression, supporting a revised $\mathrm{A}_{\mathrm{s}}$ model in which stem cell activity is limited to a subset of $A_{s}$ while remaining $A_{s}$ plus $A_{p r}$ and $A_{a l}$ are differentiation-committed or transiting into a committed state $e^{5,8}$. Given that dynamics of Id4 expression are poorly understood and relationship between transplantation capacity and in situ stem cell activity is contentious ${ }^{8,9}$, it remains to be determined whether ID4+ $\mathrm{A}_{\mathrm{s}}$ solely possess stem cell potential. Studies on ID4+ spermatogonia have often focused on neonates rather than adults 5,8 .

An alternative stem cell model is proposed based on in situ lineage tracing and live-cell imaging ${ }^{4}$. Namely, that stem cell potential is a dynamic property shared by most $\mathrm{A}_{\text {undiff }}$ irrespective of morphology but that gene expression dictates fate propensity. $A_{\text {undiff }}$ expressing Gfral (a co-receptor for glial cell line derived neurotrophic factor or GDNF) that are mostly $A_{s}, A_{p r}$ and some short $A_{a l}$, form an equipotent stem cell pool and transition between single cells and syncytial states ${ }^{10}$. $A_{\text {undiff }}$ expressing Neurogenin3 (Ngn3), primarily $A_{a l}$ plus few $A_{s}$ and $A_{p r}$, are differentiation-destined due to expression of RAR $\gamma$ and sensitivity to the differentiation stimulus retinoic acid $(\mathrm{RA})^{4,11}$. GFRa1- progenitors are also marked by transcription factor SOX $3^{12}$. However, during testis regeneration or upon transplantation, NGN3 + cells contribute significantly to the stem cell pool ${ }^{9}$. NGN3 $+A_{a l}$ can fragment to shorter chains or $A_{s}$ and revert to a GFRa1+ state, indicating that NGN3 + cells possess latent stem cell activity ${ }^{4}$. Further, subsets of NGN3 + cells marked by MIWI2 exhibit regenerative capacity but are dispensable for tissue homeostasis ${ }^{13}$. Whether stem cell potential is an inherent property of all $\mathrm{A}_{\text {undiff }}$ or restricted to a subset of cells remains to be confirmed. Regardless, stem cell activity in undisturbed tissue is primarily associated with the GFR $1+$ pool while remaining $\mathrm{A}_{\text {undiff }}$ are differentiation-primed.

Niche-derived stimuli and intrinsically expressed factors regulate $A_{\text {undiff }}$ self-renewal and differentiation. Supporting Sertoli cells produce GDNF, which is required for stem cell selfrenewal ${ }^{14}$. Basic fibroblast growth factor (bFGF) is derived from a variety of cells and maintains $A_{\text {undiff function in concert with }}$ GDNF $^{6}$. Importantly, GDNF and bFGF support long-term culture of $A_{\text {undiff }}{ }^{6}$. These factors promote $A_{\text {undiff }}$ self-renewal via induction of cell cycle genes plus transcription factors BCL6B, ETV5 and LHX1 ${ }^{15-18}$. Promyelocytic leukaemia zinc finger (PLZF or ZBTB16) promotes $A_{\text {undiff }}$ self-renewal and is an $A_{\text {undiff }}$ marker ${ }^{19-21}$. The transcription factor SALL4, a component of the pluripotency network of embryonic stem cells (ESCs) is expressed throughout the spermatogonial pool and essential for $A_{\text {undiff }}$ differentiation and self-renewal ${ }^{22,23}$. Other pluripotencyassociated factors such as Oct4 (Pou5f1) are expressed in $\mathrm{A}_{\text {undiff }}$ and have functional roles ${ }^{24}$. While multiple genes are involved in $A_{\text {undiff }}$ self-renewal and differentiation, our understanding of mechanisms underlying functional heterogeneity of $A_{\text {undiff }}$ is limited.

Germline stem cell identity remains contentious largely due to our inadequate understanding of $\mathrm{A}_{\text {undiff }}$ heterogeneity and limited markers to discriminate functionally distinct $A_{\text {undiff }}$ fractions. To address this heterogeneity at the molecular and functional level and shed light on spermatogonial hierarchy, we generated compound reporter mice based on Plzf and Oct4 promoters that have distinct expression patterns within adult $\mathrm{A}_{\text {undiff. }}$ These allowed us to isolate and define gene expression signatures of $\mathrm{A}_{\text {undiff }}$ fractions enriched in self-renewing and differentiation-primed cells. Based on this data and single cell analysis, we characterize $A_{\text {undiff }}$ heterogeneity and identify a population within the GFRal+ selfrenewing pool marked by transcription factor PDX1 plus embryonic patterning genes. We find that PDX1 selectively marks

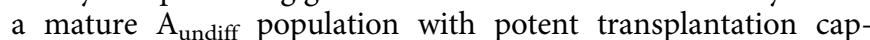
abilities and demonstrate an instructive role for niche factors in regulating interconversion between PDX1+ and PDX1- $\mathrm{A}_{\text {undiff. }}$. Our studies indicate that $A_{\text {undiff }}$ exist in a series of functionally distinct interconvertible states differentially stabilized through niche interactions.

\section{Results}

Generation of a Plzf reporter marking $\mathbf{A}_{\text {undiff. Gene expression }}$ patterns characterizing $A_{\text {undiff }}$ subsets remain poorly defined due to lack of markers allowing efficient $A_{\text {undiff isolation. To develop a }}$ system for $A_{\text {undiff }}$ purification, we generated transgenic mice expressing mCherry plus tamoxifen-regulated Cre from regulatory elements of Plzf (Plzf-mC/CreER) (Supplementary Fig. 1a $)^{19-21}$. Immunofluorescence (IF) of testis sections demonstrated that PLZF+ spermatogonia were marked with mCherry in adults from two founders (Fig. 1a and Supplementary Fig. 1b). Similar to endogenous $P l z f^{20}$, mCherry was prominent in $\mathrm{A}_{\text {undiff }}$ and differentiating type-A spermatogonia, identified according to expression of Plzf and Kit plus seminiferous epithelium cycle stage (Fig. 1a, b) ${ }^{22}$. mCherry was also detected in c-KIT + spermatogonia at late differentiation stages $(\mathrm{In} / \mathrm{B})$ in which endogenous Plzf is downregulated (Fig. 1b). Increased stability of mCherry vs. PLZF or omission of regulatory elements 
in the transgene may account for this discrepancy. Analysis of fixed, permeabilized testis cells by flow cytometry demonstrated that $\sim 90 \%$ of $\mathrm{A}_{\text {undiff }}(\mathrm{PLZF}+\mathrm{c}-\mathrm{KIT}-$ ) and essentially all early differentiating cells (PLZF $+\mathrm{c}-\mathrm{KIT}+$ ) expressed Plzf-mC/CreER (Fig. 1c and Supplementary Fig. 1c). Plzf-mC/CreER is an effective marker of undifferentiated and differentiating spermatogonia.

To confirm that Plzf-mC/CreER is expressed in stem cells, we crossed mice with a transgenic line expressing GFP upon Cre-mediated recombination (Z/EG) ${ }^{25}$. Tamoxifen (TAM) treatment of Plzf-mC/CreER; Z/EG adults induced GFP in mCherry+ spermatogonia within the epithelium basal layer by day 3 posttreatment (D3) (Fig. 1d, e). Some spermatocytes with low mCherry expression were also GFP + . At later timepoints, GFP was expressed by mature spermatocytes then spermatids as progeny of transgene-expressing cells differentiated. The majority of tubule cross-sections contained GFP+ spermatogonia D90 after TAM $(71.8 \% \pm 1.06, n=4$ testes) (Fig. 1e). Given renewal of the seminiferous epithelium (one round of spermatogenesis takes $\sim 35$ days), retention of lineage-marked cells demonstrated that Plzf-mC/CreER was active in stem cells. Whole-mount IF demonstrated that GFP was induced and maintained in a substantial fraction of GFRa1+ $A_{s}$ and $A_{p r}$, consistent with stem cell expression (Fig. 1f) ${ }^{10}$. By flow cytometry $\sim 50 \%$ of $A_{\text {undiff }}$ (PLZF+ c-KIT-) were lineage-marked over the time course, indicating transgene activity in a stable self-renewing population (Fig. 1g and Supplementary Fig. 1d). Early differentiating cells a

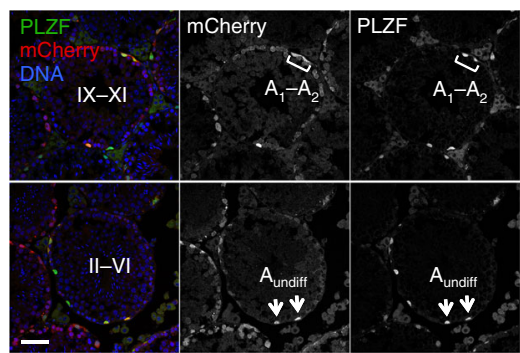

b

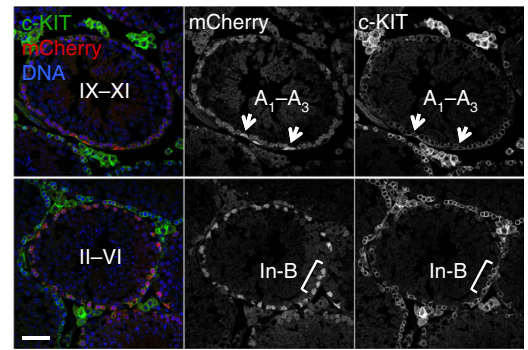

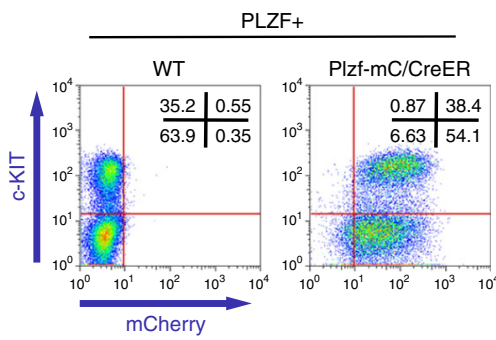

d

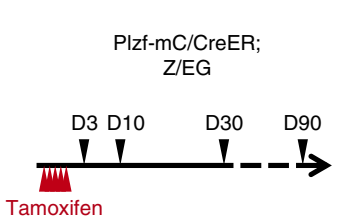

e

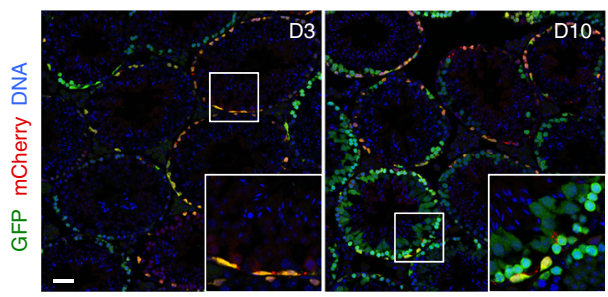

g

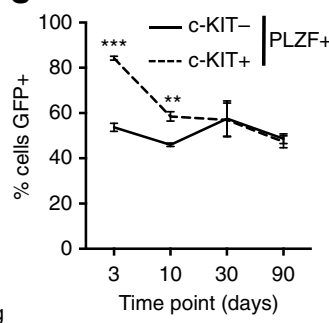

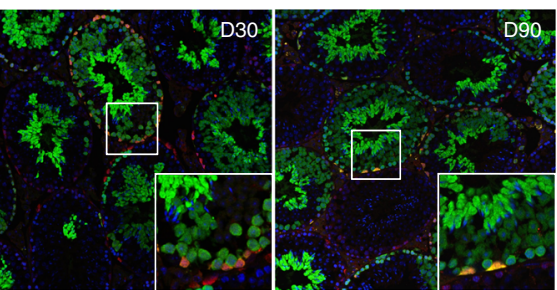
h PIz-mC/CreER

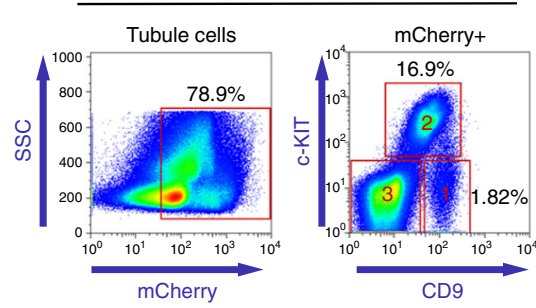

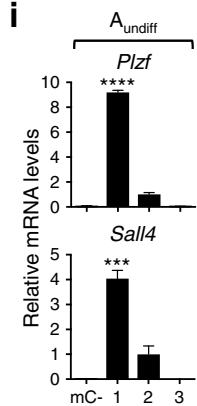
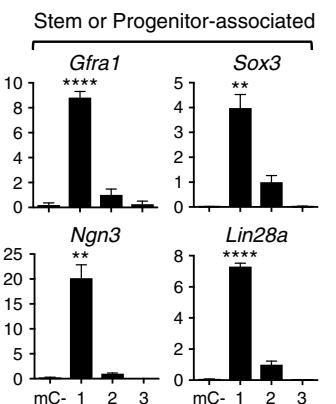

Cell population
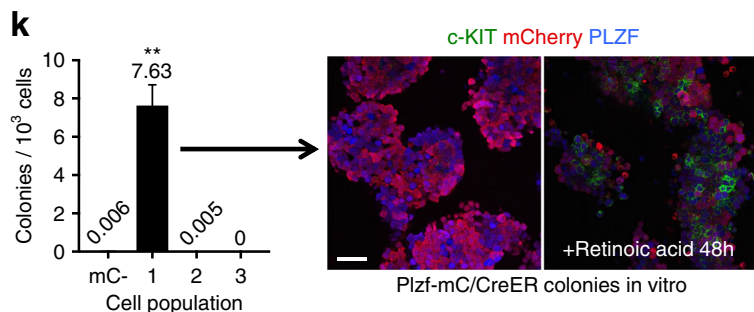

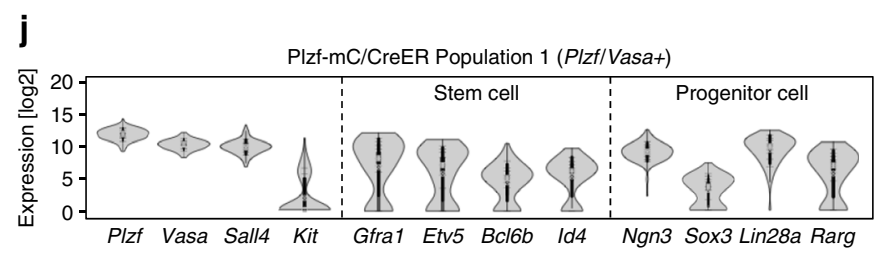

I

PIzf-mC/CreER
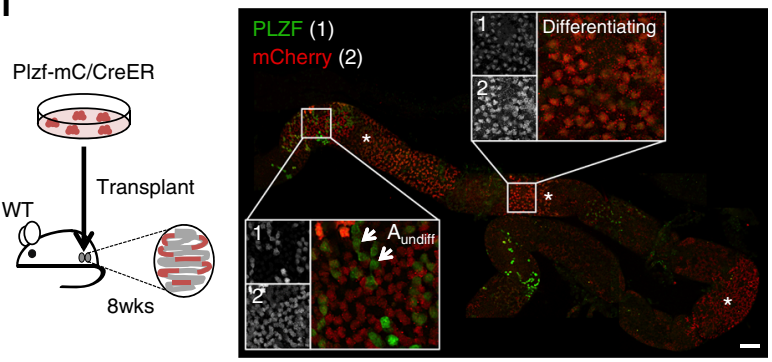
$(\mathrm{PLZF}+\mathrm{c}-\mathrm{KIT}+)$ labelled more efficiently than $\mathrm{A}_{\text {undiff }}$ at initial time points, reflecting prominent transgene expression in these cells (Fig. 1c), progression of marked cells to later differentiation stages and generation of new differentiating cohorts by labelled A undiff.

To isolate $A_{\text {undiff }}$ from germ cells marked by Plzf-mC/CreER, we stained cells for CD9 and c-KIT, markers of stem and differentiating spermatogonia, respectively ${ }^{3,26}$. CD9 is also expressed by somatic cells but selection according to CD9 and Plzf-mC/CreER was predicted to allow $\mathrm{A}_{\text {undiff }}$ isolation while excluding somatic contaminants. mCherry+ cells could be divided into three fractions: CD9+ c-KIT $-(\# 1)$, CD9+ c-KIT $+(\# 2)$ and CD9 ${ }^{\text {low }}$ c-KIT- (\#3) (Fig. 1h). Subset \#1 expresses

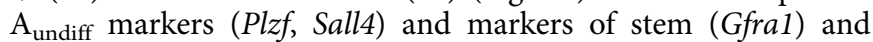
progenitor (Ngn3, Sox3, Lin28a) cells and corresponds to the $\mathrm{A}_{\text {undiff }}$ population (Fig. 1i). Subset \#2 expresses Kit and Stra8, suggesting it contains differentiating spermatogonia and early spermatocytes ${ }^{3,27}$. Cells in fraction \#3 were presumably mature spermatocytes that contained low mCherry levels (Fig. 1a, b). Selection of cells with high mCherry excluded population \#3 while \#1 and \#2 contained cells with a range of mCherry expression (Supplementary Fig. 1e). $A_{\text {undiff }}$ could not be isolated using CD9 and c-KIT alone due to contamination with Plzf-mC/ CreER - somatic cells (Supplementary Fig. 1f).

To confirm identity and purity of this putative $A_{\text {undiff }}$ fraction, we analysed gene expression of population \#1 at a single cell level by qRT-PCR (Fig. 1j). 150 out of 152 cells were positive for expression of $P l z f$ and germ cell marker Vasa, indicating somatic cell exclusion and $\mathrm{A}_{\text {undiff }}$ enrichment. These cells homogenously expressed Sall4 while Kit expression was limited to few cells. Stem cell-associated genes Gfra1, Etv5, Bcl6b and Id4 exhibited bimodal expression, consistent with heterogeneous expression in $\mathrm{A}_{\text {undiff }}$. Progenitor-associated genes Sox3 and Rarg also displayed bimodal expression patterns. The ability to generate stable clusters of cells in vitro was essentially limited to fraction \#1 and cultured cells expressed PLZF, GFRa1 and mCherry, resembling $\mathrm{A}_{\text {undiff }}$ (Fig. $1 \mathrm{k}$ and Supplementary Fig. $\left.1 \mathrm{~g}\right)^{20}$. Cultured cells upregulated c-KIT plus STRA8 and downregulated $\mathrm{A}_{\text {undiff }}$ markers in response to RA, confirming differentiation competence (Fig. $1 \mathrm{k}$ and Supplementary Fig. 1g) ${ }^{24}$. Cultures generated colonies upon transplantation, confirming stem cell activity (Fig. 11). Through development of a $\mathrm{Plzf}$ reporter, we therefore established a system for $\mathrm{A}_{\text {undiff }}$ isolation.

Pluripotency reporters exhibit distinct expression in adult $\mathbf{A}_{\text {undiff. }}$ To isolate functionally distinct $\mathrm{A}_{\text {undiff }}$ and characterize heterogeneity, we developed compound reporter mice with Plzf$\mathrm{mC} / \mathrm{CreER}$, which is expressed throughout the $\mathrm{A}_{\text {undiff }}$ pool, and
GFP-based reporters marking discrete subsets. Pluripotency gene reporters are associated with stem cell activity and potentially exhibit heterogeneous expression within $\mathrm{A}_{\text {undiff }}{ }^{28,29}$. Sox2 ${ }^{\mathrm{GFP}}$ marks transplantable testis cells and lineage-marked cells of Sox $2^{\text {CreER }}$ adults generate long-lived clones, demonstrating stem cell expression ${ }^{28}$. Restriction of Sox $2^{\text {GFP }}$ expression to isolated spermatogonia in sections suggested that Sox $2^{\mathrm{GFP}}$ marks $\mathrm{A}_{\mathrm{s}}$ and short $\mathrm{A}_{\text {undiff }}{ }^{28}$. However, whole-mount IF demonstrated that Plzf$\mathrm{mC} / \mathrm{CreER}$ and Sox $2^{\mathrm{GFP}}$ were co-expressed throughout the $\mathrm{A}_{\text {undiff }}$ pool and in c-KIT+ differentiating spermatogonia (Fig. 2a). Oct4 reporters mark transplantable cells in neonatal testis, suggesting expression in stem cells $s^{30,31}$. The transgene here is based on a 18 $\mathrm{kb}$ Oct4 gene fragment lacking proximal enhancer sequences and displays heterogeneous expression in cultured $\mathrm{A}_{\text {undiff }}{ }^{29,32}$. Wholemount IF of adult Plzf-mC/CreER; Oct4-GFP tubules indicated that Oct4-GFP expression was limited to a subset of Plzf-mC/ CreER+ spermatogonia (Fig. 2b). Surprisingly, while Oct4-GFP was expressed in A-type differentiating spermatogonia and $\mathrm{A}_{\mathrm{al}}$, it was generally absent from Plzf-mC/CreER $+\mathrm{A}_{\mathrm{s}}$ and $\mathrm{A}_{\mathrm{pr}}$ (Fig. 2b). Analysis of $\mathrm{A}_{\text {undiff }}$ by intracellular staining and flow cytometry confirmed heterogeneous Oct4-GFP expression (Fig. 2c). 40\% of $\mathrm{PLZF}+\mathrm{c}-\mathrm{KIT}-\mathrm{A}_{\text {undiff }}$ and $\sim 90 \%$ of PLZF $+\mathrm{c}-\mathrm{KIT}+$ were Oct4$\mathrm{GFP}+$ (Fig. 2d), revealing a correlation between Oct4-GFP and differentiation.

In agreement with these results, GFR $1+\mathrm{A}_{\mathrm{s}}$ and $\mathrm{A}_{\mathrm{pr}}$ (steadystate stem cells ${ }^{10}$ ) were generally Oct4-GFP- (Fig. 2e, f). In contrast, most SOX $3+$ progenitors (including $\mathrm{A}_{\mathrm{al}}$ and $\mathrm{A}_{1} / \mathrm{A}_{2}$ ) were Oct4-GFP+ (Fig. 2e, f). We confirmed that Oct4-GFP was preferentially expressed in SOX3+ progenitors vs. GFRa1+ $\mathrm{A}_{\text {undiff }}$ at all stages of the epithelium cycle (Supplementary Fig. 2a, b). Oct4-GFP expression positively correlated with spermatogonial chain length in both populations (Supplementary Fig. 2c). By comparison to markers of progenitors (RAR $\gamma$, LIN28A) and differentiating cells (DNMT3A, CCND1) ${ }^{3,20}$, we confirmed that Oct4-GFP was expressed in $\mathrm{A}_{\mathrm{al}}$ and differentiating A-type cells and downregulated at later differentiation stages (Fig. $2 \mathrm{f}, \mathrm{g}$ ).

Gonocytes of postnatal day 2 (PND2) testes were Oct4-GFP+ as anticipated, confirming transgene integrity (Supplementary Fig. 2d) ${ }^{32}$. By PND5 Oct4-GFP was heterogeneously expressed in PLZF+ spermatogonia and GFRa1+ cells had lower Oct4-GFP expression than GFRa1- cells suggesting that Oct4-GFP expression is suppressed as gonocytes migrate into the niche. OCT4 protein was restricted to Oct4-GFP+ cells in the neonate (Supplementary Fig. 2d), indicating that Oct4-GFP recapitulates aspects of endogenous Oct4 expression.

To test function of Oct4-GFP+ and Oct4-GFP- $\mathrm{A}_{\text {undiff, cells }}$ from Oct4-GFP; Plzf-mC/CreER adults were transplanted and mCherry + colonies scored after 8 weeks (Fig. $2 \mathrm{~h}$ ). While stem

Fig. 1 Characterization of Plzf-mC/CreER transgenic mice. $\mathbf{a}$, $\mathbf{b}$ Representative IF of adult Plzf-mC/CreER testis sections ( $n=3$ mice). Tubule stages and populations are indicated. Scale bar, $50 \mu \mathrm{m}$. c Representative flow cytometry of fixed and permeabilized testis from Plzf-mC/CreER and wildtype (WT) adult testis ( $n=3$ mice per genotype). PLZF+ cells are shown. $\mathbf{d}$ Plzf-mC/CreER; Z/EG mice injected daily with TAM for 5 days were harvested at indicated days after treatment. e Representative IF of testis sections from $\mathbf{d}$ ( $n=3$ testes per time point). Insets show details of indicated areas. Scale bar, $50 \mu \mathrm{m}$. f Representative whole-mount IF from d. Inset shows detail of indicated area. Arrowheads: unlabelled GFR $1+$ cells. Scale bar, $50 \mu \mathrm{m}$. $\mathbf{g}$ Flow cytometry of fixed and permeabilized testis cells from $\mathbf{d}$. Graph indicates mean fraction of $A_{\text {undiff }}$ (PLZF+c-KIT-) and PLZF+c-KIT+ early differentiating cells expressing GFP \pm standard error of mean (s.e.m.) ( $n=4$ testes D3, D10 and D90, $n=6$ testes D30). h Representative flow cytometry of live Plzf-mC/ CreER testis cells. SSC is side scatter. mCherry+ gate was set according to WT. i Quantitative RT-PCR for spermatogonial markers from Plzf-mC/CreER cell fractions sorted as in $\mathbf{h}$. $\mathrm{mC}$ - indicates $\mathrm{mCherry}-$. Expression levels are corrected to $\beta$-actin and normalized so mean value of fraction \#2 equals 1 . Mean values \pm s.e.m. are indicated ( $n=3$ sorts, 2 mice pooled per sort). Significance vs. mCherry - cells is shown. $\mathbf{j}$ Violin plots of gene expression in 150 single cells of fraction \#1 cells from $\mathbf{h}$. Cells were gated according to Plzf and Vasa expression. $\mathbf{k}$ Left: mean in vitro colony-forming activity of Plzf-mC/ CreER fractions \pm s.e.m. isolated as in $\mathbf{h}(n=3$ mice). $\mathrm{mC}$ - indicates mCherry - . Significance vs. mCherry - fraction is indicated. Right: representative IF of passaged cells from fraction \#1 treated with vehicle or retinoic acid for $48 \mathrm{~h}(n=3)$. Scale bar, $50 \mu \mathrm{m}$. I Left: transplantation of cultured cells established from Plzf-mC/CreER fraction \#1. Right: representative whole-mount IF of tubules 8 weeks post transplant demonstrating formation of mCherry + colonies $\left(n=5\right.$ recipients). Comparable spermatogenic capacity was observed upon transplantation of an independent line $\left(3.90\right.$ colonies $/ 10^{5}$ cells; $n=4$ recipient testes). Scale bar, $100 \mu \mathrm{m}$. Significance was calculated by two-tailed Student's $t$-test $\left({ }^{\star \star} P<0.01,{ }^{\star \star \star} P<0.001,{ }^{\star \star \star \star} P<0.0001\right)$ 
a

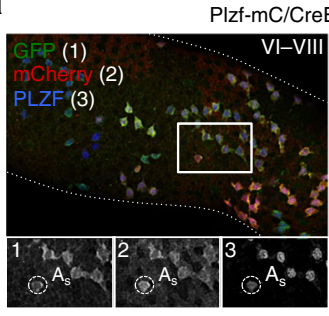

C

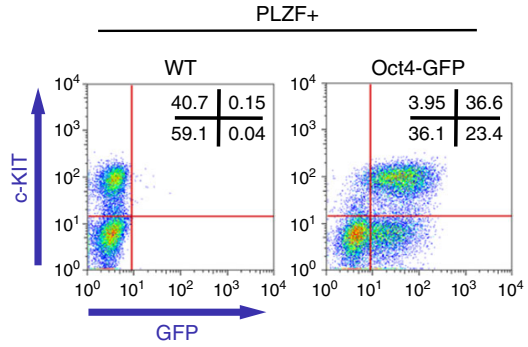

d
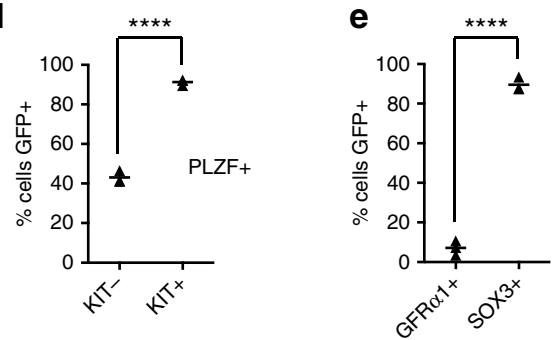

g

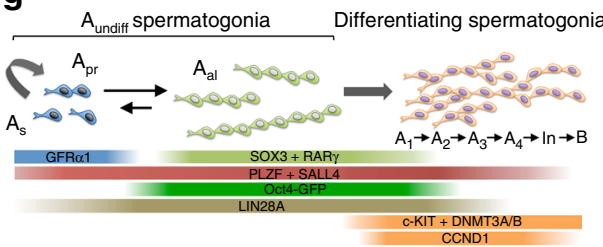

h

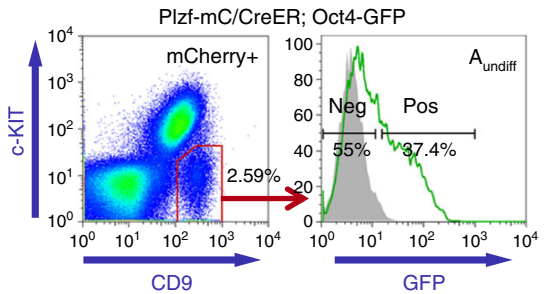

b

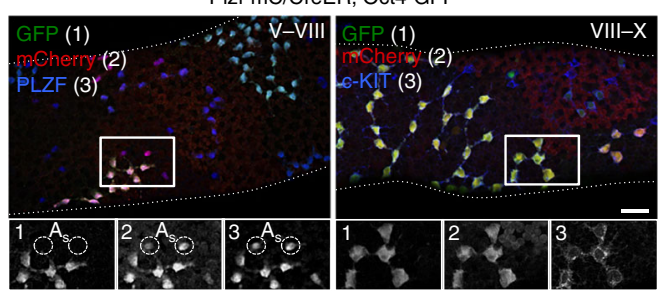

f

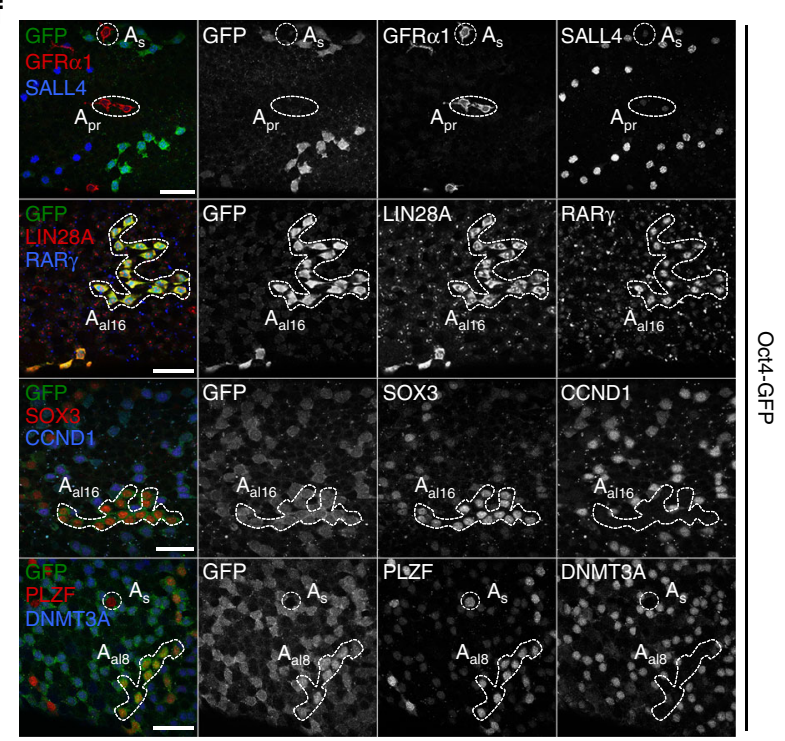

i

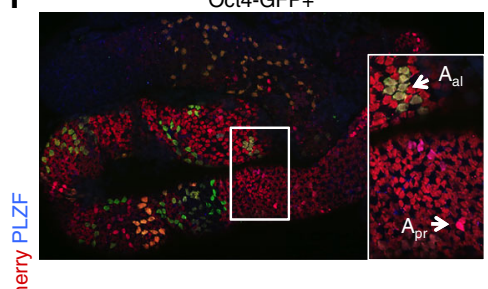

Oct4-GFP-
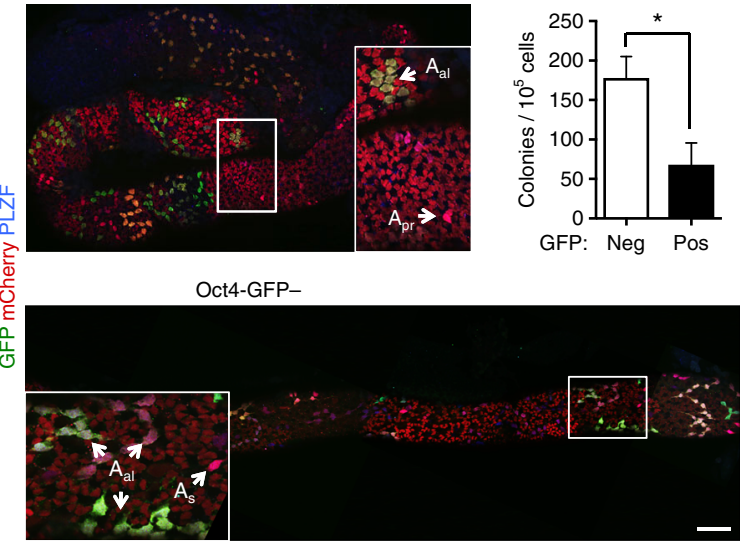

Fig. 2 Comparative analysis of reporter gene expression in spermatogonia. a, b Representative whole-mount IF of adult (8-10 weeks post natal) Plzf-mC/ CreER; Sox2 ${ }^{\text {GFP }}$ (a) and Plzf-mC/CreER; Oct4-GFP (b) seminiferous tubules. Inset panels show individual immunostaining within indicated area at higher magnification. Tubule staging and select $A_{s}$ and $A_{p r}$ are indicated. Scale bars, $50 \mu \mathrm{m}$. c Representative flow cytometry analysis of fixed and permeabilized testis cells from 1 of 3 Oct4-GFP and wild-type (WT) control adults. PLZF+ cell population is shown. Percentages of cells contained within gates are indicated. d Quantification of flow cytometry results from c. Graph indicates percentage of $A_{\text {undiff }}$ (PLZF+ c-KIT-) and cells initiating differentiation (PLZF+ c-KIT+) expressing GFP in Oct4-GFP adults. Horizontal bars indicate mean values ( $n=3$ mice). e Graph shows percentage of GFR $1+$ and SOX3+ spermatogonia positive for GFP in whole-mount seminiferous tubules of Oct4-GFP adults. Spermatogonial identity was confirmed by SALL4 counterstain. Horizontal bars indicate mean values ( $n=3$ mice, $>200$ cells scored per data point). f Representative whole-mount IF of adult Oct4-GFP seminiferous tubules for indicated markers $\left(n=3\right.$ mice). Select $A_{\text {undiff }}$ cells are indicated. Scale bar, $50 \mu \mathrm{m}$. $\mathbf{g}$ Scheme summarizing expression patterns of indicated genes and transgenic reporters plus changes in cell morphology during spermatogonial differentiation. Markers used to isolate different spermatogonial populations are indicated. $\mathbf{h}$ Isolation of Oct4-GFP - and Oct4-GFP+ $A_{\text {undiff }}$ from Plzf-mC/CreER; Oct4-GFP adults by flow cytometry. Percentage of cells in each gate from a representative sample is indicated ( $n=6$ mice). $\mathbf{i}$ Oct4-GFP - and GFP+ adult $A_{\text {undiff }}$ fractions were transplanted into recipients and analysed 8 weeks later by whole-mount IF. Images show GFP and mCherry expression in representative colonies. PLZF counterstain confirms $A_{\text {undiff }}$ and spermatogonial identity. Panels show higher magnification details of indicated areas. Scale bar, $100 \mu \mathrm{m}$. Graph shows colony-forming efficiency of Oct4-GFP+ and GFP- $A_{u n d i f f}$ fractions. Data is presented as mean number of colonies per $10^{5}$ donor cells \pm s.e.m. $(n=7$ recipient testes for Oct4-GFP- cells and $n=6$ for Oct4-GFP+ cells). Donor cells were pooled from 2 Plzf-mC/CreER; Oct4-GFP adults. Significance was calculated by two-tailed Student's $t$-test $\left({ }^{\star} P<0.05, \star \star \star \star P<0.0001\right)$ 
cell activity was significantly enriched within the Oct4-GFP$\mathrm{A}_{\text {undiff fraction }}$ as expected, the Oct4-GFP+ population also contained transplantation activity (Fig. 2i). Oct4-GFP therefore delineates $\mathrm{A}_{\text {undiff }}$ populations with distinct characteristics and stem cell activity but transplantation capability is not restricted to the Oct4-GFP- population ${ }^{9,13}$.

Gene expression signatures of stem and progenitor $A_{\text {undiff. To }}$ gain insight into $A_{\text {undiff }}$ heterogeneity we performed gene expression profiling of Oct4-GFP - and GFP $+\mathrm{A}_{\text {undiff }}$ (Fig. 3a and Supplementary Data 1). From a panel of differentially expressed genes selected according to potential relevance for stem cell function, we confirmed gene expression signatures of Oct4-GFPand GFP $+A_{\text {undiff }}$ by qRT-PCR (Fig. 3b). Oct4-GFP $+A_{\text {undiff }}$ expressed progenitor markers (Ngn3, Rarg, Nanos3, Sohlh1, Lin28a, Stra8, Sox3) at a higher level than Oct4-GFP- $\mathrm{A}_{\text {undiff. }}$ Genes downstream GDNF and/or linked with stem cell function (Lhx1, T, Egr2, Etv5, Nanos2, Gfra1, Ret) were enriched in Oct4GFP - cells ${ }^{16,33}$. Expression of stem cell marker Id4 was modestly although not significantly enriched in GFP $-\mathrm{A}_{\text {undiff }}$ (not shown). Control genes Plzf and Vasa were comparably expressed (Fig. 3b). These data support the observed expression pattern of Oct4-GFP in $\mathrm{A}_{\text {undiff }}$ and reveal gene signatures associated with stem and differentiation-primed (progenitor) fractions. Genes associated with pluripotency and embryonic germ cells were co-enriched with Oct4 in the GFP+ progenitor fraction (Dppa2, Dppa3, Gdf3, Lin28a, Prdm14, Tdh, Utf1) (Fig. 3b and Supplementary Data 1). Expression of multiple developmental and cell regulators (Eomes, Lhx1, Pdx1, Smad6, T, Tcl1) were enriched within the $\mathrm{GFP}$ - fraction.

To confirm heterogeneous expression of genes from our screen, we analysed candidates plus known $A_{\text {undiff }}$ and germ cell markers by single cell qRT-PCR of $\mathrm{A}_{\text {undiff }}$ from Plzf-mC/CreER adults. Data were processed with viSNE, which plots cells in a two-dimensional map where proximity of cell points indicates gene expression similarity ${ }^{34}$. Projection of expression of a gene of interest in colour allows visualization of cell subsets marked by that gene (Fig. 3c and Supplementary Fig. 2e). Spermatogonia with highest expression of stem cell-associated genes including Gfral grouped top right of the viSNE map while cells with highest expression of progenitor-associated genes including Rarg and Sox3 clustered at the opposing end. Cells in between these two groups co-expressed select stem and progenitor genes suggesting they represented transitional states. Expression of genes broadly active in $\mathrm{A}_{\text {undiff }}(\mathrm{Plzf}$, Sall4, Vasa) was found throughout the plot (Fig. 3d). While expression of stem cell genes $B c l 6 b$ and $I d 4$ was most evident within the stem cell fraction, they were detected in progenitors (Fig. 3d). Pax7 was expressed sporadically (Fig. 3d) ${ }^{35}$. Expression of differentiation markers Kit and Stra8 was detected, particularly in transitional cells (Fig. 3d).

Heterogeneity was apparent within the Gfral+ stem cell population; expression of Etv 5 and $L h x 2$ overlapped extensively with Gfral, while some appeared restricted to a subset. Developmental regulators Eomes, $P d x 1, T$ and $L h x 1$ marked a subset of Gfral-expressing cells at one extremity of the viSNE map furthest from progenitors, indicating that gene expression was distinct (Fig. 3c).

Unexpectedly, Oct4 expression was present throughout the viSNE plot, contrasting with restricted expression of Oct4-GFP in progenitors (Supplementary Fig. 2e). The Oct4-GFP reporter marks Oct4 expression in the embryonic germline but lacks specific enhancer elements and is randomly integrated ${ }^{32}$. Effects of integration site on transgene activity or regulatory roles of omitted enhancers might underlie differences in Oct4-GFP and Oct4 expression. However, expression of Oct4 and other pluripotency genes were similarly enriched in Oct4-GFP $+\mathrm{A}_{\text {undiff, }}$ supporting reporter validity (Fig. 3b). Differences in analysis methods, reporter mouse backgrounds or expression of Oct4 elements retained in the Oct4-GFP reporter may underlie these discrepancies ${ }^{32}$.

To confirm expression of genes of interest including Oct4 at a single cell level, we isolated $\mathrm{A}_{\text {undiff }}$ from Plzf-mC/CreER; Oct4GFP mice for single-cell RNA-Seq. Data were processed using Monocle to organize cells in a predicted developmental trajectory (pseudotime) according to transcriptional similarities ${ }^{36}$. After quality controls we identified $\sim 3500$ germ cells. Trajectory calculations were performed in semi-supervised mode using expression of genes associated with stem (Gfral, Etv5) and progenitor (Sox3, Upp1) populations (Fig. 3c) to identify the developmental pathway. A trajectory was obtained from cells only expressing one or both stem markers to those only expressing one or both progenitor markers (Fig. 3e). Transitional cells were defined as expressing combinations of stem and progenitor markers and were distributed along the trajectory with tendency to concentrate mid-trajectory (Fig. 3e). A minor population not identified as stem or progenitor cells according to these markers were "undefined undifferentiated". Expression of progenitor genes Ngn3 and Rarg increased as cells progressed along the trajectory, indicating that it recapitulated stem to progenitor transition (Fig. 3f). Oct4 expression increased at late pseudotime in agreement with Oct4-GFP expression in progenitors (Fig. 3f). Stra8 expression increased mid-pseudotime, confirming transient induction upon stem cell commitment (Fig. 3d, f). Expression of developmental regulators Eomes, $L h x 1$ and $P d x 1$ was limited to initial trajectory stages, consistent with stem cell expression (Fig. 3c, g). T expression was below the set detection level. Id4 was expressed throughout the trajectory but with increased levels at early points indicating preferential but not exclusive stem cell expression (Fig. 3d, g). Other stem cell-associated genes expressed in early trajectory were confirmed, e.g., Nefm, Smad6 and Tcll (Fig. 3h). Examination of differentially expressed genes along the trajectory revealed three clusters corresponding to genes downregulated (stem cell-associated), upregulated (progenitor-related) or transiently upregulated across pseudotime (Supplementary Fig. 3). This latter cluster included cell cycle-related genes potentially involved in stem-progenitor transition. We therefore define gene expression signatures of distinct $A_{\text {undiff }}$ populations and dynamics of gene expression during stem-to-progenitor conversion.

Identification of a unique $\mathbf{A}_{\text {undiff }}$ population. Single cell analysis revealed a subset of $A_{\text {undiff }}$ within the GFRa1+ self-renewing pool that co-expressed $P d x 1$, Eomes, $T$ and $L h x 1$. To confirm existence of this population, we assessed $P d x 1$ expression in adults by whole-mount IF (Fig. 4a). $P d x 1$ encodes a homeodomain transcription factor with roles in pancreas development ${ }^{37}$. Nuclear PDX1 was observed in SALL4+ DNMT3A - isolated spermatogonia and two cell-chains, indicating expression in $A_{s}$ and $A_{p r}$. Flow cytometry of fixed and permeabilized testis cells demonstrated that PDX1+ cells represent a minor fraction $(\sim 15 \%)$ of PLZF+ spermatogonia and are c-KIT-, confirming $\mathrm{A}_{\text {undiff }}$ identity (Supplementary Fig. 4a, b). PDX1+ cells were present at all stages of the seminiferous epithelium and a proportion was KI67+ suggesting that PDX1 does not mark $A_{\text {undiff }}$ with unique mitotic status (Fig. 4a and Supplementary Fig. 4c) ${ }^{2}$. Consistent with single cell data, PDX1 was restricted to a subset of GFRa1+ $\mathrm{A}_{\text {undiff }}$ (Fig. 4a). $\sim 30-40 \%$ of GFR $1+\mathrm{A}_{\mathrm{s}}$ and $\mathrm{A}_{\mathrm{pr}}$ were PDX1+ while GFR $1+\mathrm{A}_{\mathrm{al}}$ were typically PDX1- (Fig. 4b). PDX1+ cells were Oct4-GFP- (Fig. 4a). In testis from $P d x 1^{\text {GFP }}$ adults $^{38}$, GFP was detected in PDX1+ $\mathrm{A}_{\mathrm{s}}$ and $\mathrm{A}_{\mathrm{pr}}$, demonstrating antibody specificity (Fig. 4a and Supplementary Fig. 4d). Importantly, the 
a

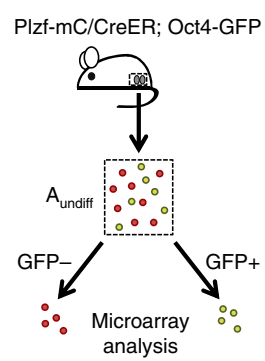

b

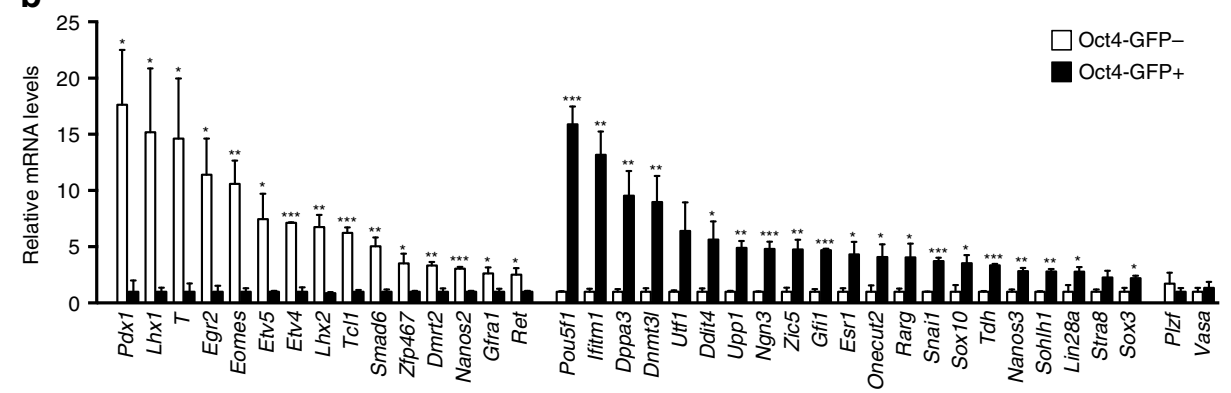

C
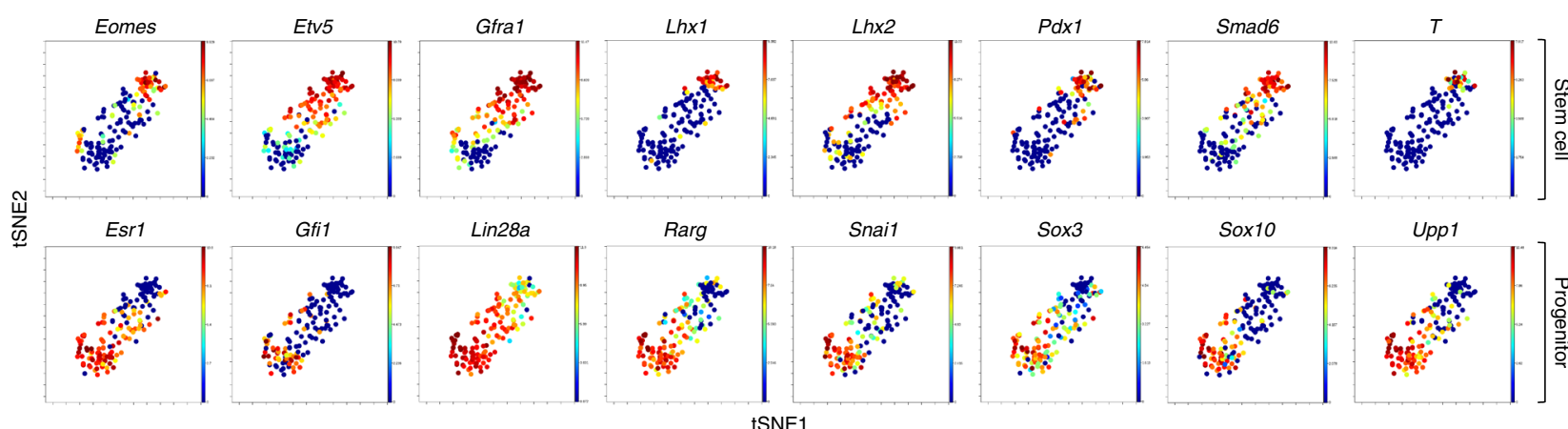

Sox3

Sox10
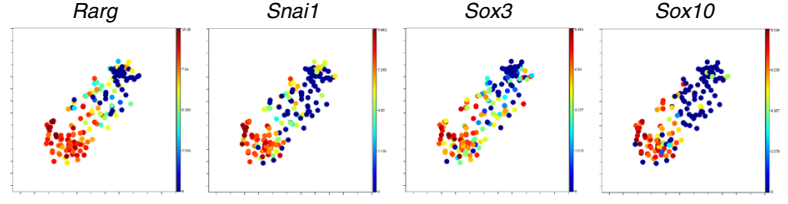

Upp1

d

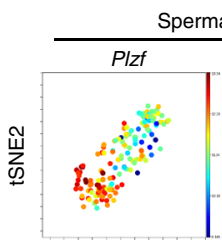

permatogonia \& germ cell markers
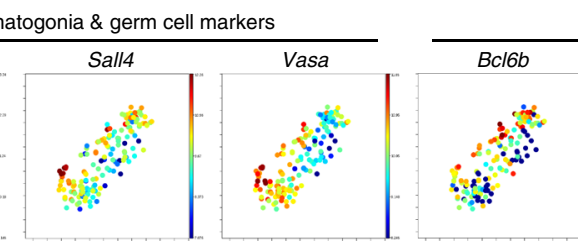

Stem cell markers
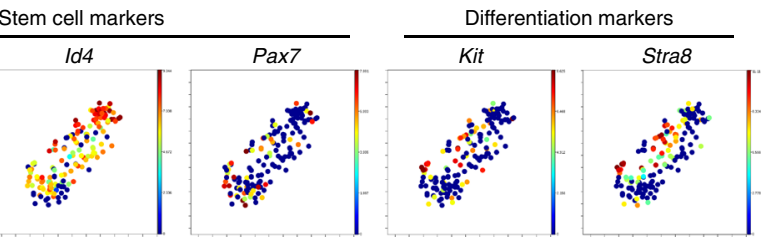

tSNE1
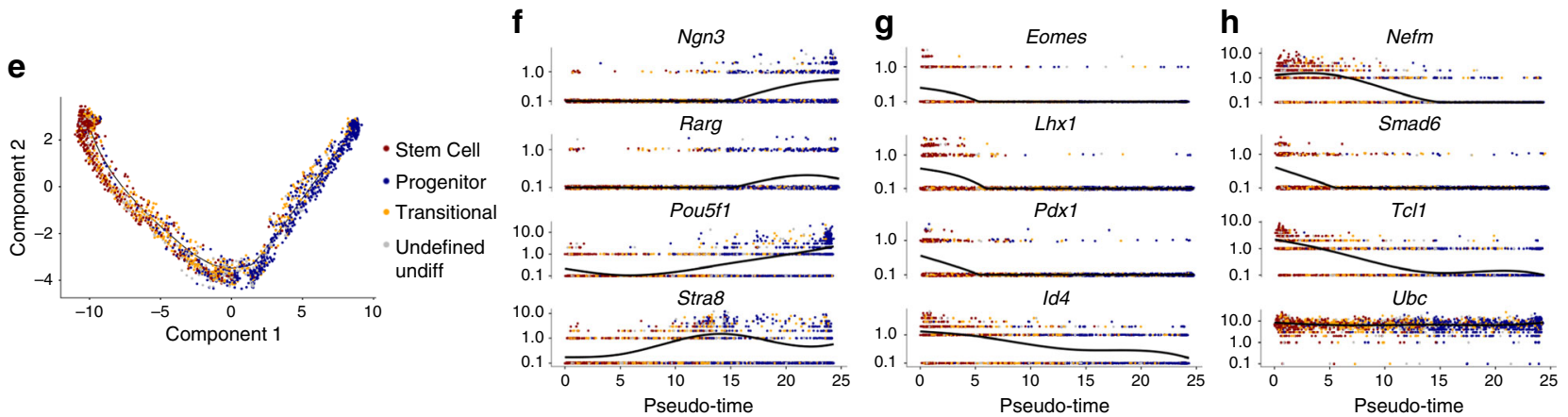

Fig. 3 Identification and characterization of distinct $A_{\text {undiff }}$ populations. a Oct4-GFP- and Oct4-GFP+ $A_{\text {undiff }}$ fractions were isolated from Plzf-mC/CreER; Oct4-GFP adults for gene expression profiling by microarray. $A_{\text {undiff }}$ fraction is mCherry+ CD9+ c-KIT-. $\mathbf{b}$ Confirmation of gene expression signatures of

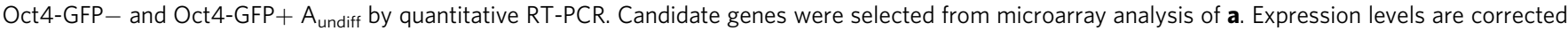
to those of $\beta$-actin and normalized so mean value of GFP- or GFP + fractions equals 1. Mean values from 3-5 mice \pm s.e.m. are indicated. Genes enriched in Oct4-GFP- and Oct4-GFP+ populations are shown in separate groups. Control genes Plzf and Vasa are shown. Significance was calculated by two-tailed Student's $t$-test $\left({ }^{\star} P<0.05,{ }^{\star \star} P<0.01,{ }^{\star \star \star} P<0.001\right)$. c viSNE maps derived from single cell analysis of 150 single $A_{\text {undiff }}$ cells isolated from pooled adult Plzf$\mathrm{mC} /$ CreER testis. Based on expression of a total of 71 genes identified from analysis of $A_{\text {undiff }}$ fractions in $\mathbf{b}$ plus genes previously linked with $A_{\text {undiff }}$ function. Relative expression of selected genes associated with stem and progenitor fractions by each plotted cell is indicated (red $=$ high, blue $=$ low). $\mathbf{d}$ viSNE maps from single cell analysis of $\mathbf{c}$. Spermatogonial and germ cell markers are in left panels. Middle panels show stem cell markers not identified as

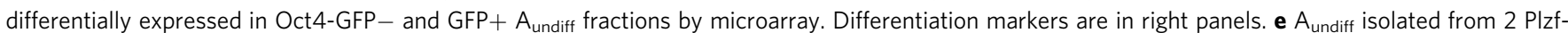
$\mathrm{mC} / \mathrm{CreER}$; Oct4-GFP adults were analysed by single cell RNA-Seq and developmental trajectory calculated using Monocle. Cells were classified as follows: Stem cells (Gfra1 or Etv5 positive), progenitors (Sox3 or Upp1 positive), transitional cells (positive for combinations of stem and progenitor genes) and undefined undiff (negative for these stem and progenitor genes). $\mathbf{f}-\mathbf{h}$ Expression of indicated genes across cell trajectory (pseudotime) from single cell analysis of $A_{\text {undiff }}$ in $\mathbf{e}$. Expression of housekeeping gene $U b c$ is shown

Plzf-mC/CreER reporter was active in PDX1+ cells in a lineagetracing setting (Fig. 4c).

We next sought to confirm additional markers of PDX1+ $\mathrm{A}_{\text {undiff. }}$ While LHX1 and T antibodies performed inadequately, we detected the T-box transcription factor EOMES in $\sim 50 \%$ of GFR $\alpha 1+$ spermatogonia in adult testis sections (Fig. $4 \mathrm{~d}$ and Supplementary Fig. 4e). By flow cytometry, EOMES+ cells were within the PLZF+ $c$-KIT $-\mathrm{A}_{\text {undiff }}$ fraction and comprised $\sim 15 \%$ 
of PLZF+ spermatogonia (Supplementary Fig. 4f, g). EOMES+ cells were Oct4-GFP - and lineage-marked in Plzf-mC/CreER; Z/ EG mice upon TAM (Fig. 4e and Supplementary Fig. 4h). Flow cytometry confirmed that PDX1+ and EOMES + cells were only present within the Oct4-GFP - fraction of the PLZF+ population (Fig. 4f). While PDX1+ spermatogonia were frequently EOMES ,$+ P d x 1$ and Eomes expression did not overlap completely; 60-
$65 \%$ of PDX1+ cells were EOMES + and vice versa (Fig. $4 \mathrm{~g}, \mathrm{~h}$ ). That PDX1 and EOMES delineated overlapping but distinct populations was evident from mitotic status. While percentage of PDX1+ cells positive for proliferation marker KI67 was similar to the bulk $\mathrm{A}_{\text {undiff }}$ pool, a higher proportion of EOMES+ cells were $\mathrm{KI} 67+$ (Fig. 4i, j). Both cell types were present throughout the seminiferous epithelium cycle but found more frequently at early a

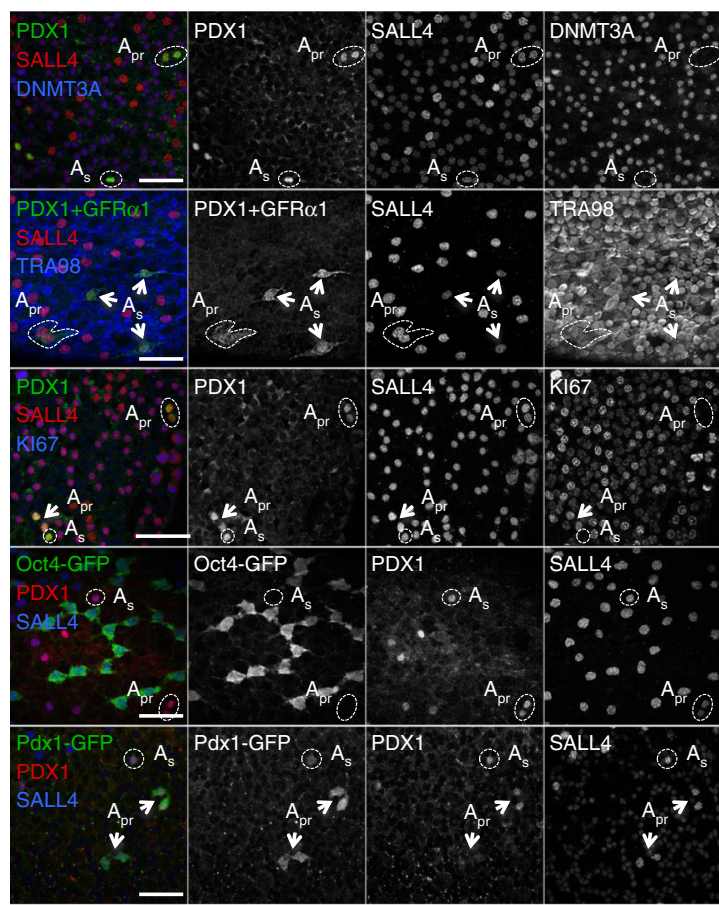

b
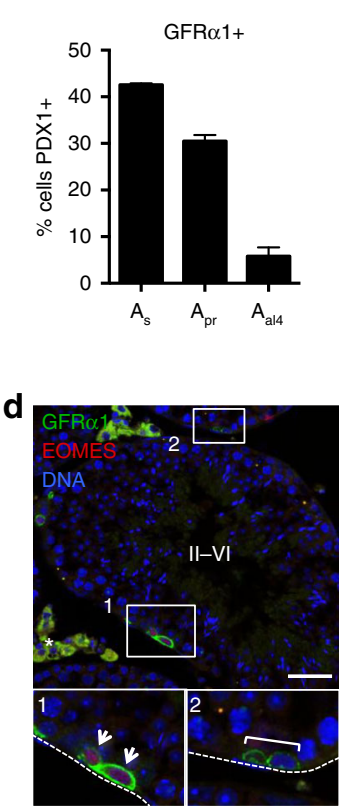
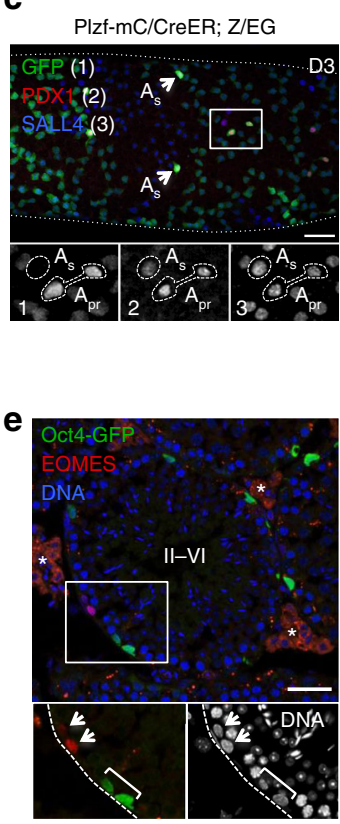

f

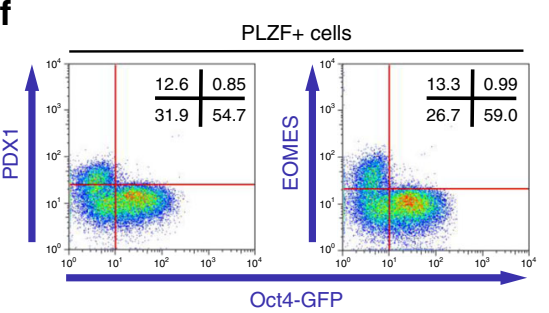

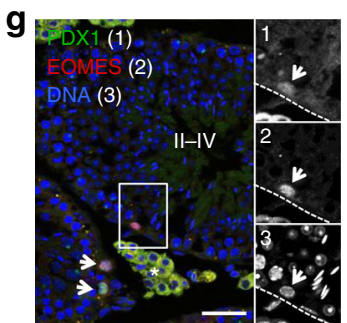

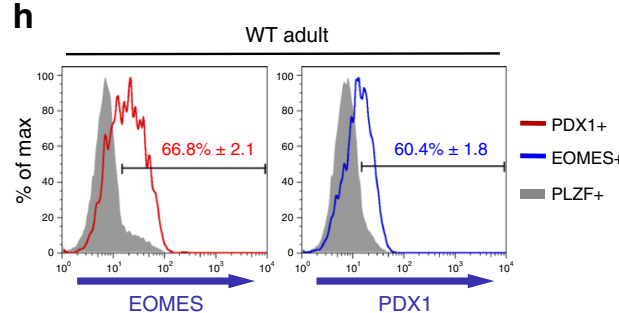

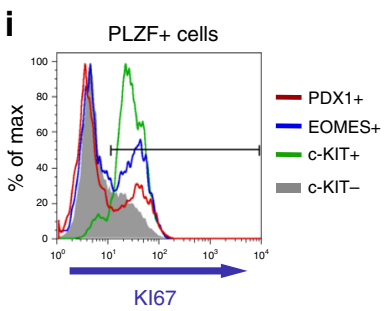

m

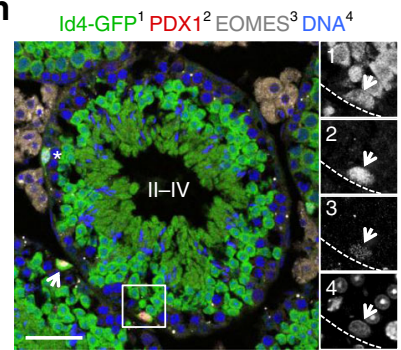

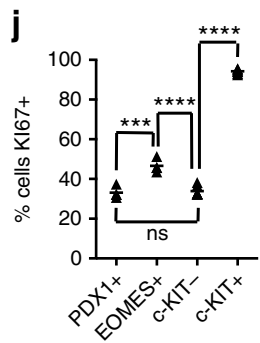

$\mathbf{k}$

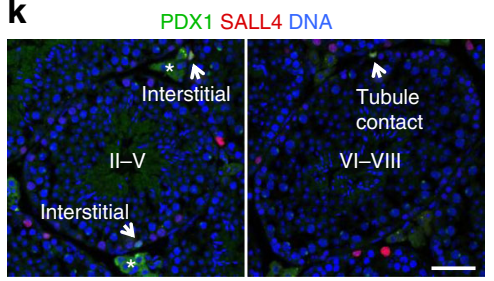

I

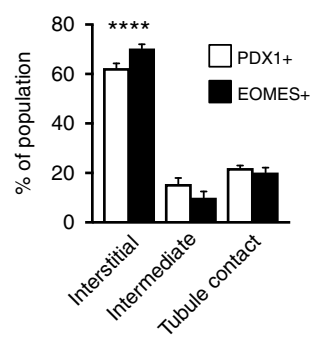

$\mathbf{n}$

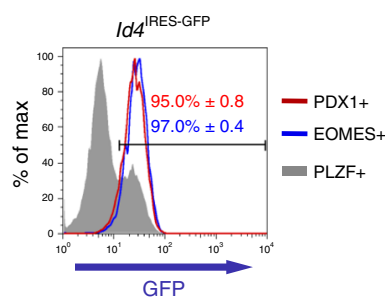

$\mathbf{0}$

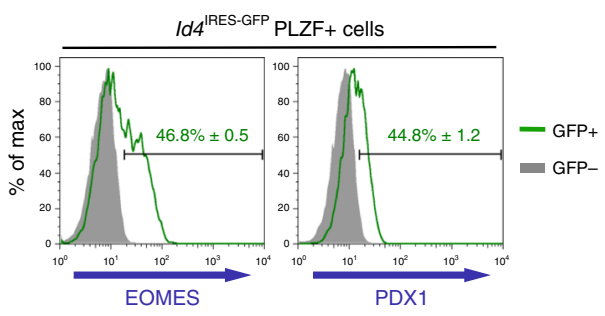


stages (Supplementary Fig. 4i). Moreover, both populations localised preferentially to tubule regions adjacent to the interstitium (Fig. 4k, l) ${ }^{39}$.

To confirm the immature nature of PDX1+ and EOMES+ $\mathrm{A}_{\text {undiff }}$ we assessed expression of stem cell marker Id4 in this population using Id $4^{\mathrm{IRES}-\mathrm{GFP}}$ mice ${ }^{5,40}$. Id4 reporter expression in adults was prominent in spermatocytes plus spermatids and marked occasional spermatogonia (Fig. $4 \mathrm{~m}$ and Supplementary Fig. 4j). While most GFP + cells were PLZF- c-KIT - by flow cytometry (spermatocytes and spermatids) a subset were PLZF+ c-KIT - ( $\left.\mathrm{A}_{\text {undiff }}\right)$ (Supplementary Fig. 4k). Approximately $30 \%$ of PLZF + spermatogonia expressed Id ${ }^{\text {IRES-GFP }}$ (Supplementary Fig. 4l, m). Spermatogonia co-expressing Pdx1, Eomes and Id4 were observed in sections but not all ID4+ spermatogonia were PDX1+ or EOMES+ (Fig. 4m and Supplementary Fig. 4j). By flow cytometry, essentially all PDX1+ and EOMES + cells were ID $4+$ but only $\sim 45 \%$ of ID4+ spermatogonia were PDX1+ or EOMES + (Fig. 4n, o), confirming more widespread expression of $I d 4$ than reported ${ }^{5}$. Combined, we identify Id4-expressing $\mathrm{A}_{\text {undiff }}$ marked by PDX1 and EOMES within the GFRa1+ stem cell pool.

Functional and molecular features of PDX1+ spermatogonia. $P d x 1$ expression is detected in GFR $\alpha 1+\mathrm{A}_{\mathrm{s}}$ and $\mathrm{A}_{\mathrm{pr}}$, suggesting it marks stem cells. To test function of PDX1+ cells, GFP + and GFP $-\mathrm{A}_{\text {undiff }}$ from $P d x 1^{\mathrm{GFP}}$; Plzf-mC/CreER mice were transplanted and mCherry+ colonies scored 8 weeks later. A small

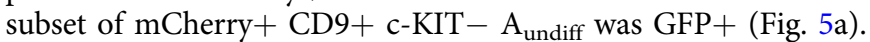
While both GFP + and GFP - fractions generated colonies, PDX1 + cells exhibited significantly higher colony-forming activity than PDX1- $A_{\text {undiff }}$ (Fig. 5b, c). Stem cell potential is therefore substantially enriched in but not exclusive to the PDX1+ population. Colony size from PDX1+ and PDX1 - fractions was comparable (Fig. 5d), indicating that PDX1+ and PDX1 - stem cells are equipotent. Equivalent GFP + populations were found in colonies from both fractions, demonstrating that PDX1 $-\mathrm{A}_{\text {undiff }}$ generate PDX1+ cells upon transplantation and vice versa (Fig. 5b, e).

To define molecular features of PDX1+ cells, we isolated GFP

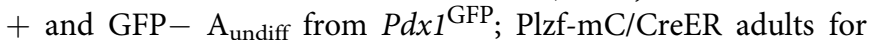
RNA-Seq. Expression of stem cell-associated genes identified from previous analysis (Fig. 3b), including Egr2, Eomes, Etv5, Gfra1, Lhx1, Lhx2, Pdx1, Smad6, T and Tcl1 were enriched in the PDX1+ population (Fig. 5f). Genes associated with progenitors including Dppa3, Esr1, Lin28a, Ngn3, Rarg, Sohlh1, Sox3, Sox10 and Upp1 were expressed at higher levels in PDX1 - cells (Fig. 5f). Control genes Plzf, Sall4, and Vasa were comparably expressed while Id4 was expressed at a higher level in PDX1+ cells. Wnt pathway inhibitor Shisa6 was enriched in the PDX1+ fraction ${ }^{41}$. Oct4 was not differentially expressed between PDX1+ and PDX1 - fractions, consistent with discrepancies between endogenous Oct4 and Oct4-GFP expression.

KEGG pathway analysis was performed on genes differentially expressed between PDX1+ and PDX1- $\mathrm{A}_{\text {undiff }}$ (Supplementary Data 2). Significant pathways included protein synthesis (ribosome, ribosome biogenesis), metabolism (OXPHOS) and cellular signalling (MAPK and NOTCH pathways) (Supplementary Fig. 5a). PDX1 + cells expressed lower levels of ribosome subunits than PDX1- $A_{\text {undiff }}$ (Supplementary Fig. 5b), consistent with a need to limit protein synthesis rates ${ }^{42}$. MAPK pathway components (Map2k2/Mek2, Map3k3/Mekk3, Mapk9/Jnk2 and Mapk11/p38b) plus MAPK regulators (Hras, Nf1, Dusp2, Dusp4, Dusp5, Dusp6 and Dusp7) were differentially expressed in PDX1 + and PDX1- $A_{\text {undiff }}$ (Fig. 5g), suggesting a central role for this pathway in $\mathrm{A}_{\text {undiff }}$ regulation ${ }^{15,43}$.

The $P d x 1^{\mathrm{GFP}}$ reporter disrupts $P d x 1$ expression due to GFP insertion $^{38}$. $P d x 1$ loss is fatal due to defective pancreas development, so the reporter was maintained in heterozygosity ${ }^{44}$. Given reported haploinsufficiency of $P d x 1^{45}$ and reduced $P d x 1$ expression in $P d x 1^{\mathrm{GFP} /+} \mathrm{A}_{\text {undiff }}$ (Fig. 5h and Supplementary Fig. 5c, d), we assessed whether germline maintenance was disrupted in $P d x 1^{\mathrm{GFP} /+}$ adults. No disruptions to PLZF+ and differentiating $c$-KIT + populations were evident in aged $P d x 1^{\mathrm{GFP} /+}$ mice (Fig. 5i and Supplementary Fig. 5e). Expression of stem cell-associated genes Eomes and Gfral in $A_{\text {undiff }}$ from $P d x 1^{\mathrm{GFP} /+}$ adults was not perturbed (Fig. 5h). PDX1 therefore marks $A_{\text {undiff }}$ with unique molecular features and potent stem cell capabilities but reduction in $P d x 1$ expression does not compromise stem cell function.

Niche factors control transitions between $\mathbf{A}_{\text {undiff }}$ states. To examine hierarchy of $\mathrm{A}_{\text {undiff }}$ fractions and mechanisms controlling fate, we used our compound reporter models and culture system. When stem and progenitor-enriched $A_{\text {undiff }}$ fractions were isolated from Plzf-mC/CreER; Oct4-GFP adults according to GFP and placed in culture, comparable numbers of cell clusters were formed (Fig. 6a, b). Cultures derived from Oct4-GFP- and $\mathrm{GFP}+\mathrm{A}_{\text {undiff }}$ had similar growth rates (Fig. 6c). Established lines

Fig. 4 Characterization of PDX1+ spermatogonia. a Representative whole-mount IF of WT (top 3 rows, $n=3$ mice), Oct4-GFP and Pdx 1 GFP adult tubules ( $n$ $=2$ mice per genotype). Antibodies to PDX1 and GFR 1 are goat polyclonals and distinguished by nuclear vs. cell surface staining respectively. Scale bars, $50 \mu \mathrm{m}$. b Mean percentage of GFR $1+$ cells/chains PDX1+ \pm s.e.m. from WT analysis in a $(n=4$ mice, $>250$ cells/chains per sample). c Representative whole-mount IF of adult PIzf-mC/CreER; Z/EG tubules D3 post-TAM ( $n=2$ mice). Arrowheads: PDX1+ $A_{s}$. Inset shows detail of indicated area. Scale bar, $50 \mu \mathrm{m}$. d Representative IF of adult WT testis sections ( $n=4$ mice). Insets: details of selected areas. EOMES + GFR $1+$ (arrowheads) and EOMES - GFR $\alpha 1$ + cells (bracket) are shown. Tubule stage is indicated. Scale bar, $50 \mu \mathrm{m}$. e Representative IF of adult Oct4-GFP testis section ( $n=2$ mice). Insets: immunostaining within indicated area. EOMES + GFP - (arrowheads) and EOMES- GFP+ (bracket) spermatogonia are indicated. Asterisks: autofluorescent interstitium. Scale bar, $50 \mu \mathrm{m}$. $\mathbf{f}$ Representative flow cytometry of fixed and permeabilized testis cells from Oct4-GFP adults $(n=2)$. PLZF+ cells shown. $\mathbf{g}$ Representative IF of adult WT testis sections $(n=4$ mice). Insets: immunostaining within indicated area. PDX1+ EOMES+ spermatogonia are indicated (arrowheads). Tubule stage is shown. Asterisk: autofluorescent interstitium. Scale bar, $50 \mu \mathrm{m}$. h Representative flow cytometry of fixed and permeabilized WT adult testis cells $(n=4)$. Mean numbers of EOMES + and PDX1+ cells in PDX1+ and EOMES+ gates respectively are shown \pm s.e.m. $i$ Representative flow cytometry for KI67 in indicated PLZF+ populations of fixed and permeabilized adult WT testis cells ( $n=4$ mice). $\mathbf{j}$ Quantification of flow cytometry from i. Percentage of indicated PLZF+ fractions KI67+. Horizontal bars: mean values ( $n=4$ mice). $\mathbf{k}$ Representative IF of adult WT testis sections demonstrating PDX1+ $\mathrm{A}_{\text {undiff }}$ localisation (arrowheads) within tubules ( $n=4$ mice). Asterisks: autofluorescent interstitium. Tubule stages are shown. Scale bar, $50 \mu \mathrm{m}$. I Quantification of spermatogonial localisation from k. Mean values \pm s.e.m. are shown ( $n=4$ mice, $56-95$ tubule sections per mouse). Significance vs. tubule-tubule localisation is indicated. $\mathbf{m}$ Representative IF of Id4 IRES-GFP adult testis sections ( $n=4$ mice). Insets show immunostaining within indicated area. Arrowheads: PDX1+ EOMES+ GFP+ spermatogonia. Asterisk: PDX1 ${ }^{\text {low }}$ EOMES + GFP+ cell. Tubule stage is shown. Scale bar, $50 \mu \mathrm{m}$. $\mathbf{n}$ Representative flow cytometry of indicated PLZF+ populations of fixed and permeabilized adult Id 4 IRES-GFP testis cells $(n=4$ mice). Mean numbers of PDX1+ and EOMES $+A_{\text {undiff }}$ expressing GFP \pm s.e.m. o Flow cytometry of Id4 ${ }^{\text {IRES-GFP }}$ testis cells as in $\mathbf{n}$. PLZF+ fractions are shown. Mean numbers of PLZF+ GFP+ cells positive for EOMES and PDX1 are indicated \pm s.e.m. $(n=4$ mice). Significance was calculated by two-tailed Student's $t$-test $\left({ }^{\star \star \star} P<0.001,{ }^{\star \star \star \star} P<0.0001\right)$ 
were positive for $A_{\text {undiff }}$ markers (PLZF, SALL4, GFRa1) plus mCherry and expressed Oct4-GFP heterogeneously (Fig. 6a). Our data suggested that cultures can be initiated from self-renewing and differentiation-primed $A_{\text {undiff }}$ and cells readily interconvert between stem and progenitor states in vitro (Fig. 2i). Cultures from both sources generated colonies upon transplantation, confirming stem cell capacity (Fig. 6d and Supplementary Fig. 6a).

To confirm dynamics of $\mathrm{A}_{\text {undiff }}$ interconversion, sorted Oct4GFP - and GFP + cells from established lines were plated at defined density and GFP expression monitored (Fig. 6e). The majority of Oct4-GFP+ cells transitioned into a GFP - state within 1 week but GFP+ cells accumulated at later points. Oct4GFP - cells generated GFP+ populations over a 2-week period. Adoption of a GFP+ state correlated with increasing culture density (Supplementary Fig. 6b).

Gene expression signatures of freshly isolated Oct4-GFP- and GFP $+A_{\text {undiff }}$ were mostly retained in fractions from cultures, indicating that Oct4-GFP marks similar populations in vitro and in vivo (Fig. 6f). Importantly, expression of stem cell-associated markers Egr2, Eomes, Etv5, Lhx2, $P d x 1$ and $T$ were enriched in Oct4-GFP - cells in vitro. However, $L h x 1$ became evenly expressed a

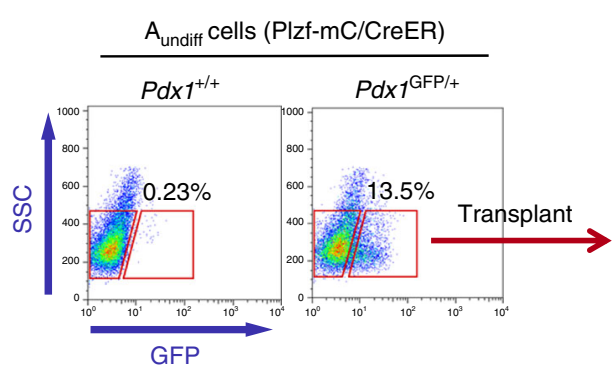

b

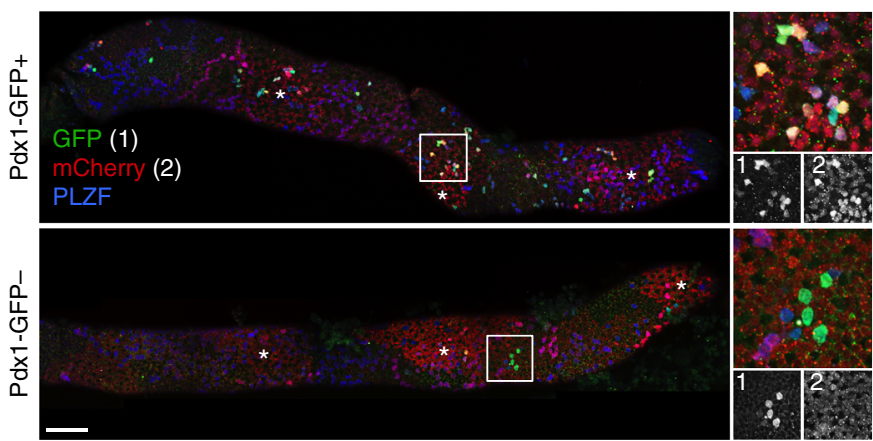

C

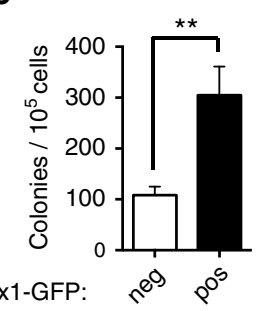

g

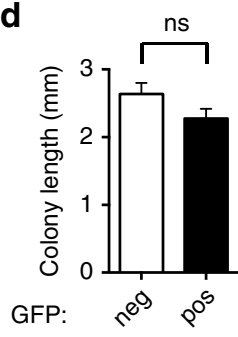

d

GFP: $\quad r^{89} p^{0^{5}}$ e

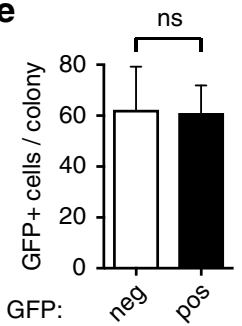

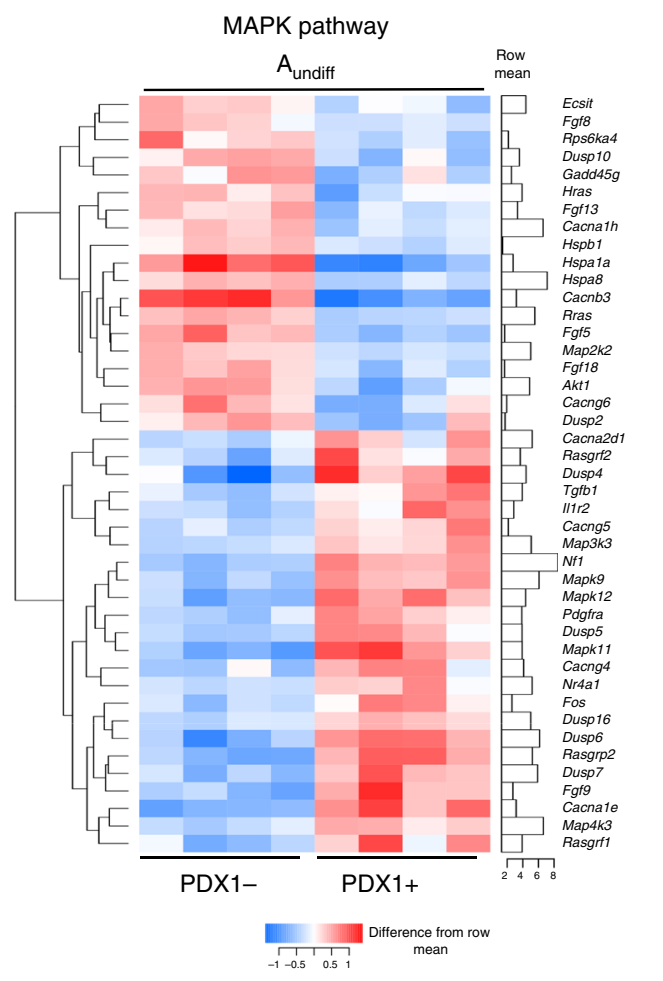

h
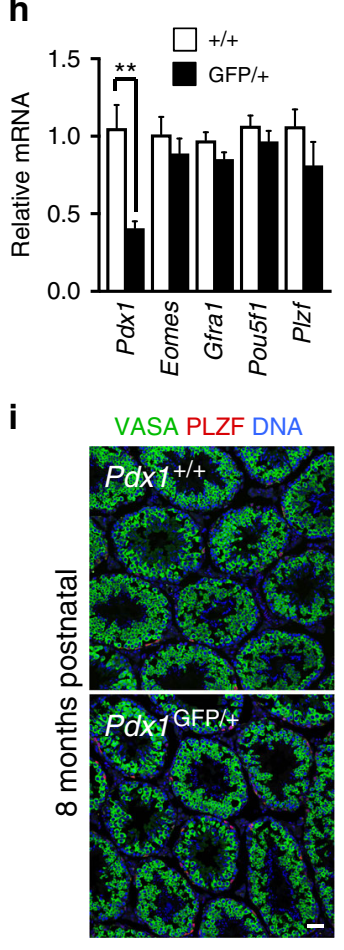

f

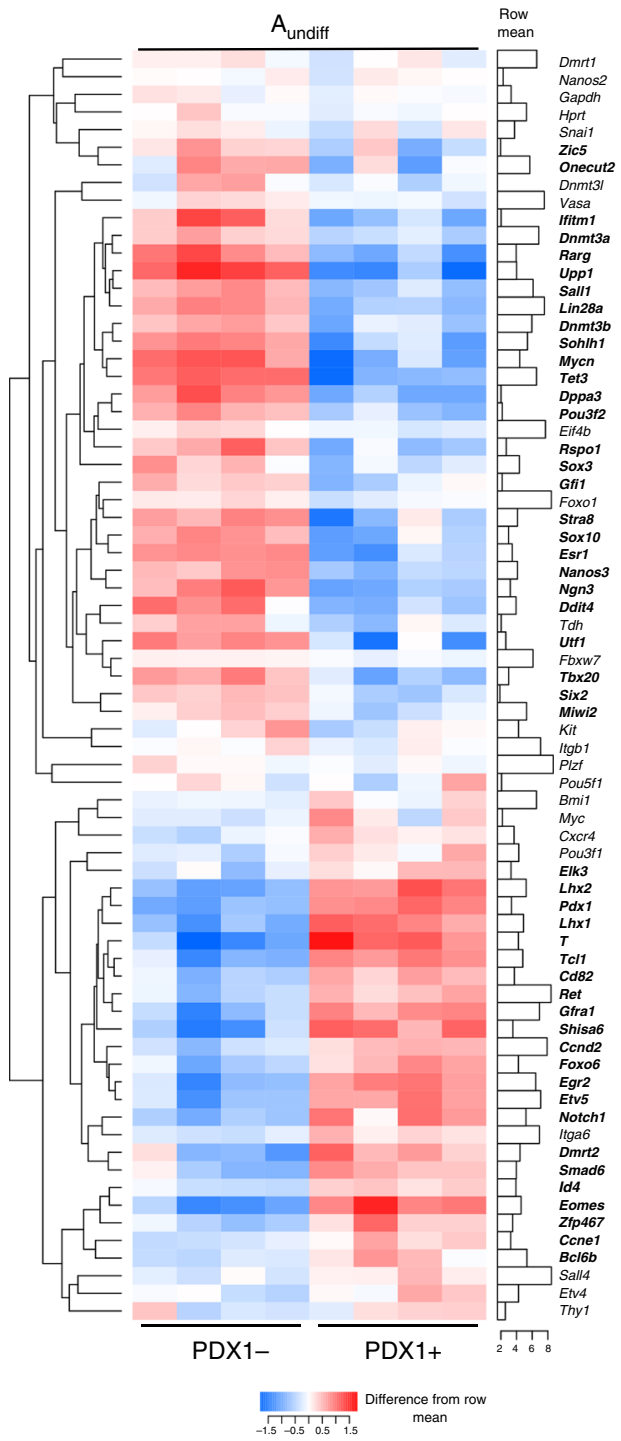


between Oct4-GFP - and GFP + fractions in culture (Fig. 6f). To confirm abundance of $\mathrm{A}_{\text {undiff }}$ fractions during culture, we compared $P d x 1$ and Oct4-GFP expression upon passaging (Fig. 6g). Cell clusters formed from both stem and progenitor-enriched $\mathrm{A}_{\text {undiff }}$ fractions contained PDX1+ and Oct4-GFP+ cells. $P d x 1$ and Oct4GFP expression were mutually exclusive and Eomes expression mirrored that of $P d x 1$ (Supplementary Fig. 6c). Oct4-GFP+ cells dominated upon passaging while PDX1+ and EOMES + cells became relatively rare (Fig. $6 \mathrm{~g}$ and Supplementary Fig. 6c).

As cultures are founded at clonal density then passaged at higher densities, we hypothesized that declining abundance of PDX1+ and EOMES + cells upon passaging was due to highdensity culture. When plated at low density, established cultures contained prominent PDX1+ and EOMES + populations and reduced numbers of Oct4-GFP+ cells while increasing density caused accumulation of Oct4-GFP + cells and reduced PDX1+ and EOMES + populations (Fig. $6 \mathrm{~h}$ and Supplementary Fig. $6 \mathrm{~d}$ ). Importantly, GDNF was more rapidly exhausted from media of cells cultured at high vs. low density and increasing GDNF and bFGF levels reduced abundance of Oct4-GFP+ cells and c-KIT+ differentiating populations (Fig. 6i, j and Supplementary Fig. 6e).

These data suggested an instructive role for niche factors in promoting stem vs. progenitor cell states. As GDNF and bFGF regulate self-renewal via distinct pathways ${ }^{46}$, we assessed importance of each factor in maintenance of the stem cell state. From sorted Oct4-GFP+ cells of established cultures, GDNF promoted adoption of an Oct4-GFP - state while bFGF did not (Fig. 6k). Conversely, the Oct4-GFP - state of sorted cells was lost when bFGF alone was present but maintained with GDNF (Fig. 6k). Cell growth under single factor conditions was more limited than with both factors (Supplementary Fig. 6f). When maintained with bFGF alone, cultures contained more c-KIT+ cells, consistent with switch to a differentiation-primed state (Supplementary Fig. 6g). GDNF thus plays a dominant role in supporting the stem cell fraction. Cell clusters formed with GDNF alone contained more PDX1+ cells than those in media containing bFGF alone (Fig. 61). When in an appropriate environment, $A_{\text {undiff }}$ therefore reversibly transition between progenitor and stem cell states. Interconversion between discrete states is regulated by niche factors.

Consistent with the role of ERK MAPK downstream GDNF in vivo ${ }^{43}$, treatment with a MAPK pathway inhibitor depleted PDX1+ and EOMES + populations in low-density cultures and increased Oct4-GFP expression (Fig. $6 \mathrm{~m}$ and Supplementary Fig. 6h, i). Inhibitors to PI3Kinase and mTOR, both linked to $\mathrm{A}_{\text {undiff }}$ differentiation ${ }^{43,47,48}$, promoted generation of EOMES+ cells and suppressed proliferation as indicated by KI67 (Fig. $6 \mathrm{~m}$ and Supplementary Fig. 6i). Effects of PI3Kinase and mTOR inhibitors on PDX1+ populations were less pronounced (Fig. $6 \mathrm{~m}$ and Supplementary Fig. 6i), suggesting that regulatory inputs to these genes are distinct.

PDX1 and EOMES define distinct stem cell states. The nascent stem cell population is formed during the first postnatal week of testis development and expands dramatically until steady-state spermatogenesis is established ${ }^{49,50}$. PDX $1+$ cells were not found in neonatal testis (PND5) and were most evident in adults (Fig. 7a). Occasional PDX1+ cells were observed at PND10 but significant populations not present until PND20. In contrast, EOMES was detectable in subsets of GFRa1+ spermatogonia at PND10 and PND20 (Fig. 7b and Supplementary Fig. 7a). At PND10, EOMES + cells were generally PDX1 - although some were PDX $1^{\text {low }}$. EOMES + PDX $1+$ cells became evident by PND20 (Fig. 7b). Distinct developmental timelines for PDX1+ and EOMES $+\mathrm{A}_{\text {undiff }}$ were confirmed by flow cytometry (Fig. 7c, $\mathrm{d}$ and Supplementary Fig. $7 \mathrm{~b}, \mathrm{c}$ ).

$P d x 1$ induction coincides with transition from growing to steady-state tissue, indicating that PDX1 marks mature stem cells. Consistent with distinct regulatory inputs, EOMES marks stem cells of developing and mature testis although the proportion of EOMES $+\mathrm{A}_{\text {undiff }}$ increased modestly during development (Fig. 7d). The proportion of $A_{\text {undiff }}$ and EOMES+ cells that were KI67+ declined from PND10 to PND20 and adults consistent with declining mitotic status of stem cells (Supplementary Fig. $7 \mathrm{~d}-\mathrm{f})^{51}$. Pdx1 expression did not correlate with $\mathrm{A}_{\text {undiff }}$ quiescence (Fig. 4i, j), suggesting that PDX1 is not responsible for lower proliferative rates of mature stem cells.

Male germline cells are depleted by the alkylating agent busulfan. Regeneration of the seminiferous epithelium is reliant on "repopulating stem cells" resistant to genotoxic stress but whether these cells represent a specific $A_{\text {undiff }}$ subset remains unclear $^{52}$. To test whether PDX1 marks repopulating stem cells, WT mice were treated with a low busulfan dose that depletes differentiating spermatogonia and a substantial $\mathrm{A}_{\text {undiff }}$ fraction (Supplementary Fig. $7 g-i)^{4,22}$. Few spermatogonia one-week after treatment expressed $P d x 1$, suggesting that PDX1 does not mark busulfan-resistant $A_{\text {undiff }}$ (Supplementary Fig. 7j). By 2 weeks, remaining $A_{\text {undiff }}$ have initiated a regenerative response characterized by long GFRa1+ $\mathrm{A}_{\mathrm{al}}$ rarely observed in undisturbed testis (Fig. 7e $)^{4}$. These $A_{a l}$ were mitotically active as indicated by KI67 but were PDX1-. PDX1+ cells were evident in areas

\footnotetext{
Fig. 5 Stem cell potential and molecular characteristics of PDX1+ $A_{\text {undiff. }}$ a Isolation of PDX1+ and PDX1- $A_{\text {undiff }}$ from Plzf-mC/CreER; Pdx 1 GFP/+ adults by flow cytometry. $A_{\text {undiff }}$ are mCherry+ CD9+ c-KIT-. Gates for GFP+ and GFP - cells were set according to Plzf-mC/CreER control (left profile). Percentage of cells in GFP+ gate from representative sample is shown $(n=7)$. b Pdx1-GFP+ and GFP- adult $A_{\text {undiff }}$ fractions were transplanted into recipient testis and analysed 8 weeks later by whole-mount IF. Images show GFP and mCherry expression in representative donor colonies. Panels on right show higher magnification details of indicated areas and grayscale panels show individual immunostaining. Scale bar, $100 \mu \mathrm{m}$. c Colony-forming efficiency

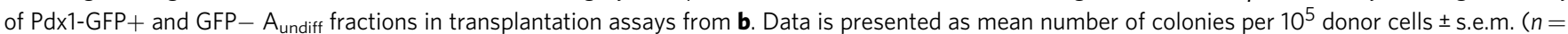
15 recipient testes for Pdx1-GFP- cells and $n=13$ for Pdx1-GFP+ cells). Donor cells were pooled from a total of 7 Plzf-mC/CreER; Pdx 1 GFP/+ adults. d Mean length \pm s.e.m. of donor colonies was measured from tiled microscope images from experiment of $\mathbf{c}$. e Mean number of GFP + cells $/$ donor colony $\pm \mathrm{s}$. e.m. were calculated for a set of whole-mount samples from transplant assay of $\mathbf{c}(n=58$ colonies from Pdx1-GFP- cells and $n=63$ from Pdx1-GFP + ). $\mathbf{f}$ Heatmap illustrates expression of indicated genes from RNA-Seq analysis of Pdx1-GFP+ and GFP- $A_{\text {undiff }}$ fractions isolated as in a ( $n=4$ mice).

Differentially expressed genes (DEG) are in bold. Cut-off for DEG is false discovery rate (FDR) $<0.05$ and absolute fold change $\geq 1.5$. $\mathbf{g}$ Heatmap showing differentially expressed MAPK pathway genes from KEGG analysis of RNA-Seq data from $\mathbf{f}$. $\mathbf{h}$ Quantitative RT-PCR analysis of indicated genes in mCherry $+\mathrm{CD} 9+\mathrm{c}-\mathrm{KIT}-\mathrm{A}_{\text {undiff }}$ isolated from Plzf-mC/CreER; $P d x 7^{\mathrm{GFP} /+}$ and Plzf-mC/CreER; $P d x 1^{+/+}$control adults. Mean values \pm s.e.m. are shown $(n=6$ mice per genotype). i Representative IF of testis sections from aged ( 8 months) mice of the indicated genotypes ( $n=3$ mice). VASA and PLZF staining identifies germ cell and spermatogonial populations respectively. Scale bar, $50 \mu \mathrm{m}$. Significance was calculated by two-tailed Student's $t$-test $\left({ }^{\star \star} P<0.01\right.$, not significant (ns) $P>0.05$ )
} 
a

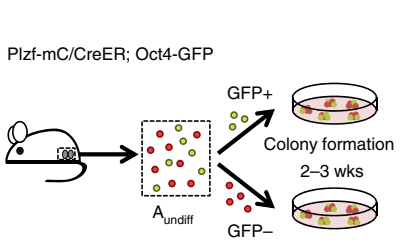

b

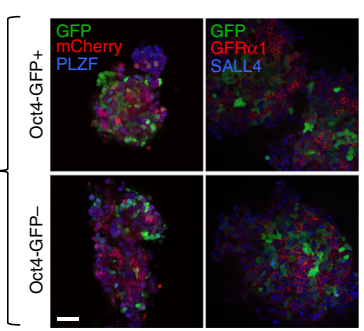

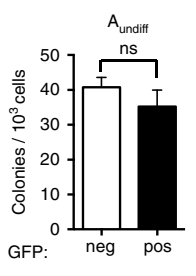

C

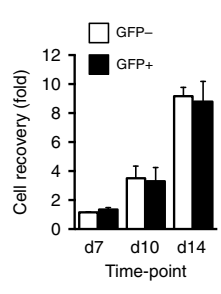

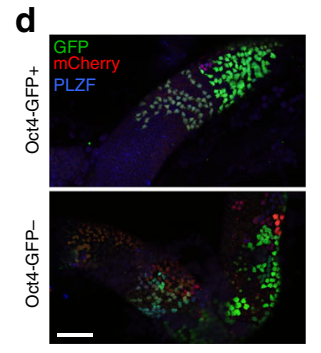

e

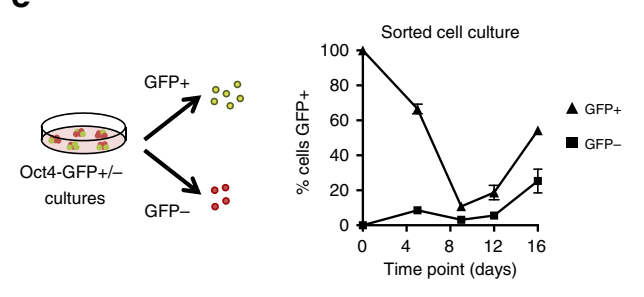

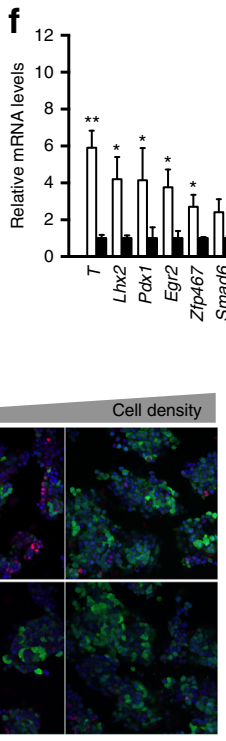

h

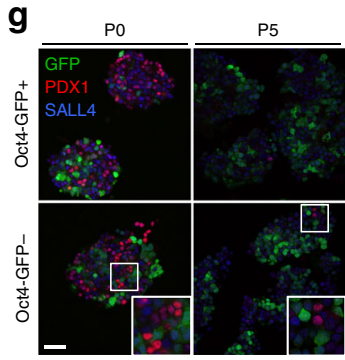

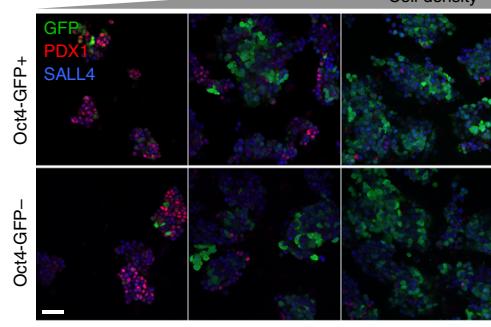

i

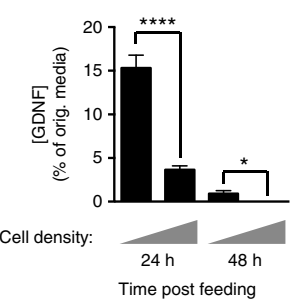

j

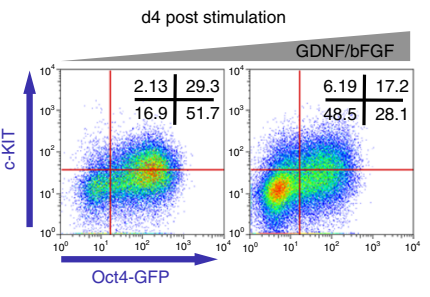

m

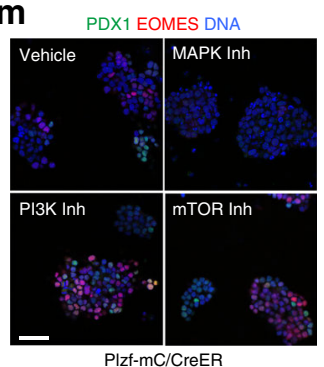

Fig. $6 \mathrm{~A}_{\text {undiff }}$ heterogeneity during culture. a Oct4-GFP - and GFP $+\mathrm{A}_{\text {undiff }}$ from Plzf-mC/CreER; Oct4-GFP adults placed in culture and analysed 2-3 weeks later. Right: IF of colonies ( $n=2$ per fraction). Scale bar, $50 \mu \mathrm{m}$. b Mean colony-forming efficiency of Oct4-GFP- and GFP $+A_{\text {undiff }} \pm$ s.e.m. from $\mathbf{a}(n=6$ mice). c Cultures from Oct4-GFP - and GFP $+A_{\text {undiff }}$ plated at $25 \times 10^{3}$ per well and counted at indicated timepoints. Mean recovery \pm s.e.m. shown. d Cultures from Oct4-GFP - and GFP $+A_{\text {undiff }}$ transplanted and analysed 8 weeks later by IF. Representative colonies shown ( 2 sets of lines) ( $n=11$ testes Oct4-GFP- and $n=9$ testes Oct4-GFP + ). Scale bar, $100 \mu \mathrm{m}$. e Cultures from Oct4-GFP- and GFP $+A_{\text {undiff }}$ sorted by GFP and plated at $25 \times 10^{3}$ per well. GFP + cells determined by flow cytometry. Mean \pm s.e.m. shown ( $n=6$ cultures). $\mathbf{f}$ qRT-PCR of GFP + and GFP- cells from Oct4-GFP- and $\mathrm{GFP}+\mathrm{A}_{\text {undiff }}$ cultures. Expression corrected to $\beta$-actin and normalized so mean of GFP- or GFP + fractions equals 1 . Mean \pm s.e.m. shown ( $n=4$ cultures). g Representative IF of primary colonies (PO) and passage 5 (P5) cultures from Oct4-GFP- and GFP $+A_{\text {undiff }}(n=4$ lines). Scale bar, $50 \mu$ m. h Cultures from Oct4-GFP - and GFP+ $A_{\text {undiff }}$ plated at increasing densities $\left(10 \times 10^{3}, 100 \times 10^{3}\right.$ and $200 \times 10^{3}$ cells/well) and analysed 7-10 days later.

Representative IF shown ( $n=4$ lines). Scale bar, $50 \mu \mathrm{m}$. i Cultures from Oct4-GFP- and GFP $+\mathrm{A}_{\text {undiff }}$ plated at low and high densities $\left(20 \times 10^{3}, 200 \times 10^{3}\right.$ cells/well) and cultured for 2 weeks. Conditioned media was collected at indicated times (hours) after media replenishment for ELISA. Mean GDNF levels are shown as percentage of starting levels \pm s.e.m. ( $n=4$ cultures). $\mathbf{j}$ Cultures from Oct4-GFP- $\mathrm{A}_{\text {undiff }}\left(20 \times 10^{3}\right.$ cells/well) switched to media containing reduced GDNF and bFGF $(1 \mathrm{ng} / \mathrm{ml}$, left) or maintained with regular media (right) for 4 days. Representative flow cytometry shown. $\mathbf{k}$ Cultures from Oct4-GFP - and GFP $+\mathrm{A}_{\text {undiff }}$ sorted according to GFP and plated $\left(10 \times 10^{3}\right.$ cells/well) in media with GDNF but no bFGF (GDNF) or bFGF without GDNF (bFGF). Cells analysed at indicated timepoints by flow cytometry. Mean percentage of cells GFP + \pm standard deviation (s.d.) ( $n=3$ replicates) from representative experiment shown. Grey plots: mean values in regular media (GDNF+ bFGF). I Cultures from Oct4-GFP- and GFP $+\mathrm{A}_{\text {undiff }}\left(20 \times 10^{3}\right.$ cells $/$ well) grown 3 days in regular media switched to media without GDNF or bFGF (-), bFGF without GDNF (bFGF), GDNF without bFGF (GDNF) or regular medium (GDNF+ bFGF) then analysed by IF after 2 weeks. Representative images shown ( $n=2$ lines). Scale bar, $50 \mu \mathrm{m}$. $\mathbf{m}$ Plzf-mC/CreER cultures incubated in media containing indicated inhibitors (Inh) for 4 days prior to IF. Representative images shown $(n=2$ lines). Scale bar, $50 \mu$ m. Two-tailed Student's $t$-test used $\left({ }^{\star} P<0.05,{ }^{\star \star} P<0.01,{ }^{\star \star \star} P<0.001,{ }^{\star \star \star \star} P<0.0001\right)$ 
a

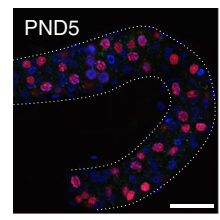

PDX1 SALL4 KI67

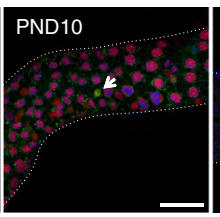

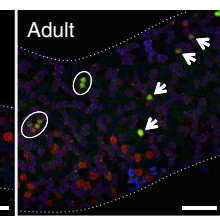

d

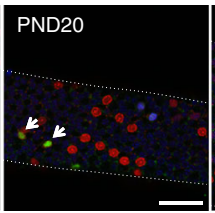

e

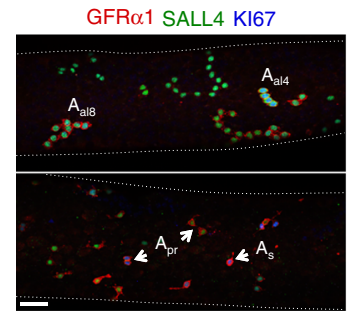

b

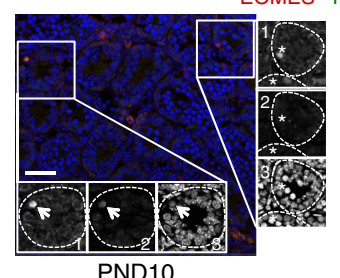

PND10

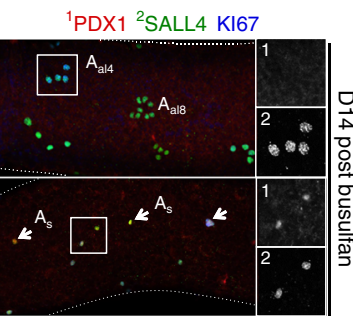

EOMES $^{1} \mathrm{PDX}^{2}{ }^{2} \mathrm{DNA}^{3}$

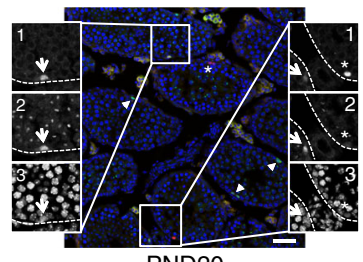

PND20

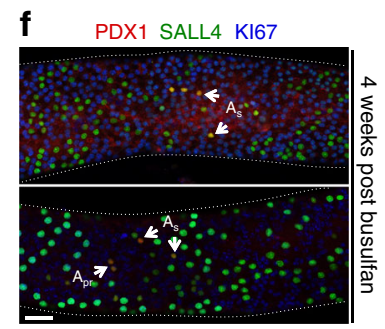

g

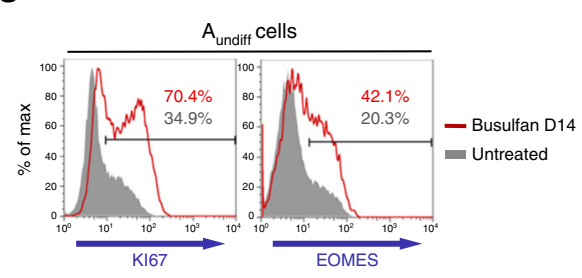

k

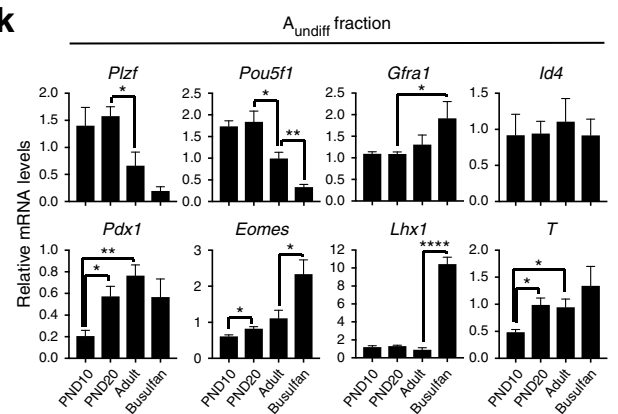

h $\square$ Untreated

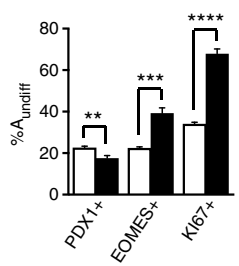

i

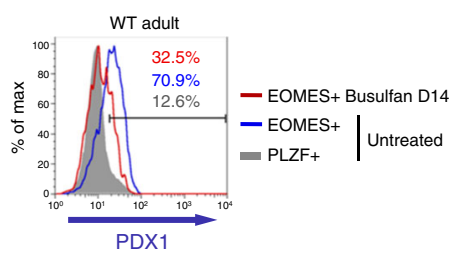

j

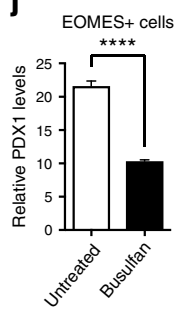

I

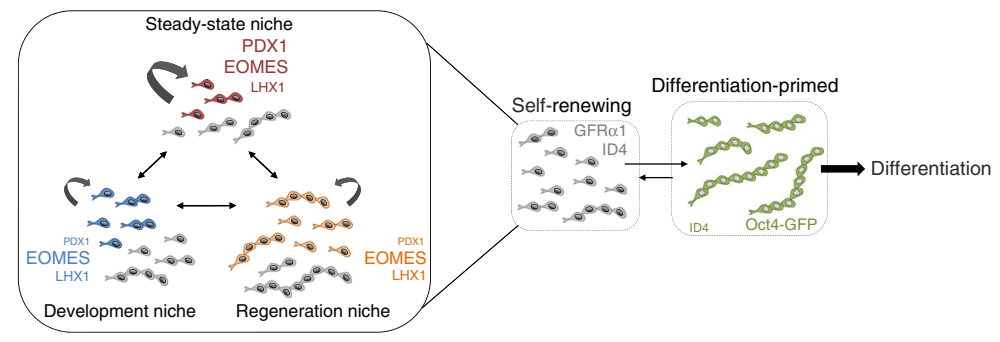

Fig. 7 PDX1+ and EOMES + spermatogonia during development and regeneration. a Representative whole-mount IF of tubules from WT mice of indicated ages (PND; postnatal day) ( $n=2$ mice per age). Scale bars, $50 \mu \mathrm{m}$. b Representative IF of testis sections from WT mice of indicated ages $(n=3$ mice per timepoint). Insets show details of indicated areas. Arrowheads: EOMES+ PDX1+ cells. Asterisks: EOMES+ PDX1- cells. Scale bars, $50 \mu \mathrm{m}$. c, d Flow cytometry of fixed and permeabilized testis cells from WT mice of indicated ages. Mean percentages of PLZF+c-KIT- $A_{\text {undiff }}$ expressing PDX1 and EOMES are shown \pm s.e.m. ( $n=3-5$ mice/time point). e WT adults were treated with busulfan $(10 \mathrm{mg} / \mathrm{kg})$ and tubules analysed by whole-mount IF 14 days later $\left(n=2\right.$ mice). Top panels: regenerative areas with GFR $\alpha 1+A_{a l}$. Bottom: non-regenerative areas lacking GFR $1+A_{a l}$. Insets show details of indicated regions. Scale bar, $50 \mu \mathrm{m}$. f Regeneration assay of $\mathbf{e} 4$ weeks post busulfan ( 2 areas shown). Arrowheads: PDX1+ $A_{\text {undiff. }}$ Scale bar, $50 \mu \mathrm{m}$. $\mathbf{g}$ Representative flow cytometry of fixed and permeabilized testis cells from WT adults untreated or busulfan treated as in $\mathbf{e}$ ( $n=4$ busulfan-treated mice and $n=5$ untreated). PLZF+ c-KIT- $A_{\text {undiff }}$ are shown. Percentage of $A_{\text {undiff }}$ KI67+ and EOMES+ are indicated. $\mathbf{h}$ Mean percentages of PLZF+ c-KIT- $A_{\text {undiff }}$ expressing PDX1, EOMES and KI67 from $\mathbf{g}$ are shown \pm s.e.m. $\mathbf{i}$ Representative flow cytometry of PDX1 in indicated populations from $\mathbf{g}$ ( $n=4$ mice per condition).

Percentages of cells PDX1+ are indicated. $\mathbf{j}$ PDX1 levels (median fluorescent intensity) in EOMES+ PLZF+ cells from i. Mean values \pm s.e.m. shown. $\mathbf{k}$ Quantitative RT-PCR of $A_{\text {undiff }}$ from Plzf-mC/CreER mice of indicated ages or 10 days post busulfan as in e. Expression levels are corrected to $\beta$-actin and normalized to an adult sample. Mean values \pm s.e.m. are indicated ( $n=4$ PND10 and busulfan-treated, $n=5$ PND20, $n=6$ adults). Selected significance values are shown. I Model of $A_{\text {undiff }}$ functional states. Self-renewing (curved arrows) GFR $\alpha 1+A_{\text {undiff }}$ adopt different states identified by variable expression

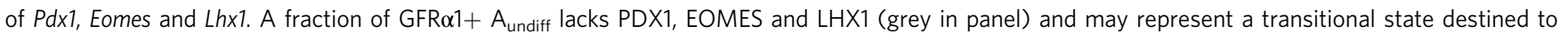
become differentiation-primed. Niche signals differentially support self-renewing states. Given dynamic niche properties, different states predominate in development and homeostatic plus regenerative testis. The state marked by PDX1, EOMES and LHX1 is specific to homeostatic testis and potentially optimized for life-long germline maintenance. $P d x 1$ is downregulated and Eomes plus $L h x 1$ upregulated in states suited for short-term expansion during development and regeneration. Oct4-GFP+ differentiation-primed $A_{\text {undiff }}$ convert back to a self-renewing state under optimised culture conditions or by transplantation into recipient testis with vacant niches. Significance was calculated by two-tailed Student's $t$-test $\left({ }^{\star} P<0.05,{ }^{\star \star} P<0.01,{ }^{\star \star \star} P<0.001\right.$, $\star \star \star \star P<0.0001)$ 
containing GFRa1+ $\mathrm{A}_{\mathrm{s}}$ and $\mathrm{A}_{\mathrm{pr}}$ but few $\mathrm{A}_{\mathrm{al}}$, which did not appear actively regenerating (Fig. 7e). At 4 weeks, tubule regions contained dense populations of $\mathrm{A}_{\text {undiff }}$ and differentiating spermatogonia, indicating successful regeneration (Fig. 7f). PDX1+ populations were found within these areas, consistent with PDX1 marking steady-state stem cells.

Analysis of $\mathrm{A}_{\text {undiff }} 2$ weeks post busulfan by flow cytometry revealed that coincident with increased mitotic activity indicated by KI67, a greater proportion were EOMES+ (Fig. 7g, h). Eomes expression was still restricted to GFRa $1+$ cells during regeneration (Supplementary Fig. 7k). While most EOMES+ $\mathrm{A}_{\text {undiff }}$ express $P d x 1$ in steady-state testis (Fig. 4h), PDX1 levels in EOMES + cells under regenerative conditions were reduced (Fig. 7i, j). Accordingly, the proportion of $A_{\text {undiff }}$ expressing $P d x 1$ was lower than in controls (Fig. $7 \mathrm{e}, \mathrm{h}$ ). Our data suggest that stem cells upregulate Eomes and downregulate $P d x 1$ under regenerative conditions. Alternatively, EOMES $+\mathrm{A}_{\text {undiff }}$ might be busulfan-resistant and downregulate $P d x 1$ during regeneration.

To confirm changes in gene expression during development and regeneration, we isolated $\mathrm{A}_{\text {undiff }}$ from Plzf-mC/CreER mice at different postnatal ages and 10 days after busulfan for qRT-PCR (Fig. 7k and Supplementary Fig. 7l, m). Across postnatal ages (PND10, 20, adult), expression of stem markers Gfra1 and Id4 were essentially constant while Gfral modestly increased after busulfan (Fig. 7k). $P d x 1$ expression was increased in $A_{\text {undiff }}$ from PND20 and adults compared to PND10 while expression was modestly although not significantly downregulated after busulfan, reflecting persistence of PDX1+ cells in some tubules (Fig. 7e). As anticipated, Eomes expression increased marginally during development but was strongly upregulated after busulfan (Fig. 7k). Other genes marking PDX1/EOMES + adult $\mathrm{A}_{\text {undiff }}$ exhibited distinct dynamics. Lhxl expression was constant during development but strikingly ( 10 fold) increased following busulfan. $T$ expression increased during development but was less responsive to busulfan. Control genes Plzf and Oct4/Pou5f1 were downregulated in adult $v s$. PND20 $\mathrm{A}_{\text {undiff }}$ and reduced following busulfan, suggesting prominent roles in developing rather than

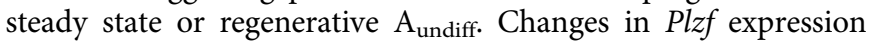
were reflected in altered activity of the Plzf-mC/CreER reporter (Supplementary Fig. 7n). Distinct combinations of Eomes, Lhx1, $P d x 1$ and $T$ expression therefore mark stem cells of developing, homeostatic and regenerating testis.

\section{Discussion}

$\mathrm{A}_{\text {undiff }}$ heterogeneity is poorly characterized and cellular hierarchy debated. Taking advantage of compound reporter mice and single cell analysis, we defined gene expression signatures associated with distinct $A_{\text {undiff }}$ populations. We confirmed that $A_{\text {undiff }}$ are broadly divided into GFR $1+$ (stem) and RAR $\gamma+$ (differentiation-primed) fractions ${ }^{10,11}$. However, gene expression was not homogenous within these populations and we identify a subset of GFRa1+ cells marked by co-expression of $P d x 1$, Eomes, Lhxl and T. Heterogeneous T expression within GFRal+ spermatogonia is reported but functional relevance incompletely understood $^{41}$. We demonstrated by transplantation that PDX1+ cells have significantly enhanced stem cell activity compared to remaining $A_{\text {undiff }}$ but that stem cells are not exclusive to this fraction. Moreover, in vivo and in vitro evidence indicates that $\mathrm{A}_{\text {undiff }}$ reversibly transition between these discrete populations and implicate niche factors in regulation of such transitions. $P d x 1$ induction within $\mathrm{A}_{\text {undiff }}$ coincided with testis maturity while other genes marking adult PDX1+ cells, e.g., Eomes, were expressed during testis development. Further, $P d x 1$, Lhx1 and Eomes displayed distinct expression dynamics during regeneration. We therefore propose a revised model of $\mathrm{A}_{\text {undiff }}$ hierarchy, whereby adult $A_{\text {undiff }}$ exist in a series of dynamic interconvertible states that variably express $P d x 1$, Eomes, Lhxl, Gfral and Oct4-GFP and have distinct functional characteristics (Fig. 7l). $A_{\text {undiff }}$ states are stabilized through interactions with a cognate niche, the property of which varies during development, regeneration and upon tissue homeostasis. Niche status dictates whether specific states, e.g., PDX1+, are sustained. Assignment of $\mathrm{A}_{\text {undiff }}$ to these distinct states would affect the balance between self-renewal and differentiation plus functional activity of the $\mathrm{A}_{\text {undiff }}$ compartment. Replenishment of cells in the differentiation-primed state and restoration of self-renewing fractions after genotoxic damage is possible via dynamic interconversion of $A_{\text {undiff }}$ states.

Our data argue against strict hierarchical organization of $A_{u n d i f f}$ fractions akin to that for ID4+ and ID4- $\mathrm{A}_{\text {undiff }}{ }^{53}$. Id4 also appears more broadly expressed in $A_{\text {undiff than }}$ proposed ${ }^{5}$, complicating interpretation of this model. Oct4-GFP+ PDX1EOMES - progenitor fractions readily generate Oct4-GFPPDX1+ EOMES + cells upon culture and Oct4-GFP $+\mathrm{A}_{\text {undiff }}$ transplant and generate $\mathrm{GFP}-\mathrm{A}_{\text {undiff }}$ despite minimal Oct4-GFP expression within the GFRa1+ self-renewing pool. Similarly, PDX1 - $\mathrm{A}_{\text {undiff }}$ generate transplantation colonies containing PDX1+ cells. Our data is consistent with a dynamic stem cell compartment in which $\mathrm{A}_{\text {undiff }}$ exhibit context-dependent stem cell activity ${ }^{4}$. The frequency of cellular transitions under physiological conditions needs to be considered. Oct4-GFP marks GFRa1-SOX3 + RAR $\gamma+\mathrm{A}_{\mathrm{al}}$, cells that co-express Ngn3 and are differentiation-destined in undisturbed testis ${ }^{19,21}$. This fate is dictated by RA sensitivity and periodic RA pulses ${ }^{11}$. Differentiation commitment is also associated with $A_{\text {undiff }}$ eviction from the niche ${ }^{39}$. Placing differentiation-primed $A_{\text {undiff }}$ in an in vitro environment lacking exogenous RA and containing supraphysiological levels of niche factors can be predicted to restore a selfrenewing state. Endogenous stem cell depletion in transplantation recipients permits access of differentiation-primed cells to the niche and an environment promoting self-renewal. Such settings are however unable to rescue self-renewal capacity once $A_{\text {undiff }}$ differentiate ${ }^{54}$. Our observations highlight a limitation of the transplant assay; $A_{\text {undiff }}$ destined to differentiate in undisturbed tissue still generate colonies in a transplant setting ${ }^{9,13}$. Lineage tracing provides better insight into cell fate in vivo but also suffers limitations ${ }^{55}$.

Interconversion of distinct states within the GFRa1+ pool evidently occurs in vivo. Formation of PDX1+ $A_{\text {undiff, }}$ presumably from GFRa1+ PDX1- cells, is observed during postnatal development. Transplanted PDX1- $\mathrm{A}_{\text {undiff }}$ generate PDX1 + cells, mimicking this developmental transition. Upon germline recovery following busulfan, the PDX1+ population is restored, potentially from GFR $1+$ EOMES + cells. GFR $\alpha 1+$ cells may routinely transit through distinct states even in steady state while Oct4-GFP+ progenitors must be removed from their physiological context to revert efficiently to a self-renewing state. Lineage tracing studies, e.g., of the GFR 1 1 + PDX1 - population, will be needed to confirm cellular transitions in situ.

Comparison of the male germline with other high-turnover tissues, e.g., haematopoietic, can provide insight into stem cell characteristics in developing and homeostatic settings. Haematopoietic stem cells (HSCs) of the foetal mouse are more mitotically active than adult counterparts and have higher self-renewal capacity ${ }^{56}$. A switch in murine HSC activity occurs 3-4 weeks postnatally, accompanied by changes in gene expression and differentiation potential ${ }^{57}$. Self-renewal mechanisms of foetal and adult HSCs are distinct and postnatal transition in HSC properties regulated through intrinsic cues ${ }^{58,59}$. While transplantable stem cells are present in early postnatal testis onwards, agedependent changes in $\mathrm{A}_{\text {undiff }}$ properties and self-renewal mechanisms are poorly appreciated. Our data indicate that 
common to HSCs, $\mathrm{A}_{\text {undiff }}$ are more mitotically active in neonates than adults. Transplantation studies demonstrated that stem cells from pups have altered proliferative capacities and differentiation tendencies than those of adults ${ }^{51} . P d x 1$ is induced in a population of $A_{s}$ and $A_{p r}$ in young adults that possess potent transplantation capabilities, suggesting that PDX1 marks the onset of a mature stem cell phenotype. After germ cell depletion by busulfan, $P d x 1$ is downregulated in regenerating $\mathrm{A}_{\text {undiff }}$ and PDX1+ populations only recover upon epithelium renewal. Similar demands are likely placed on stem cells in developing and regenerating tissues, i.e., founder/remaining stem cells expand rapidly to establish/restore the stem cell compartment then generate differentiating cells. Loss of PDX1 $+\mathrm{A}_{\text {undiff }}$ during regeneration is consistent with development of this population in homeostatic testis. It will be of great interest to characterize phenotypic and functional differences between $A_{\text {undiff }}$ under homeostatic vs. developmental or regenerative conditions and factors responsible for divergent properties.

Given that PDX1 $+\mathrm{A}_{\text {undiff }}$ development coincides with maturation of supporting Sertoli cells and GDNF promotes adoption of a PDX1 + cell state in vitro ${ }^{60}$, extrinsic cues can drive formation of this population. Genes sharing similarly restricted expression as $P d x 1$ in adult $\mathrm{A}_{\text {undiff }}$ (Lhxl, Eomes, $T$ ) are positively regulated by GDNF in cultured $A_{\text {undiff, supportive of a role for }}$ this niche factor ${ }^{16,33}$. However, GDNF levels peak in early postnatal testis and are induced following busulfan-mediated germ cell depletion ${ }^{14,61}$, conditions in which PDX1+ cells are not found. The presence of high bFGF levels in addition to GDNF may promote $A_{\text {undiff }}$ expansion under these conditions but prevent adoption of the PDX1+ state ${ }^{62}$. EOMES $+A_{\text {undiff }}$ are present during postnatal development and enriched in regenerative testis, consistent with GDNF-dependent regulation. Whether conserved signals regulate $P d x 1$ expression in foregut tissues and postnatal testis remains to be determined. Selective induction of genes such as $P d x 1$ in adult stem cells indicates that besides neonatal gonocyte-to-spermatogonia transition, stem cell formation involves an additional developmental step occurring upon exposure of nascent $\mathrm{A}_{\text {undiff }}$ to a mature testis environment.

\section{Methods}

Mouse generation and manipulation. The Plzf-mC/CreER transgenic reporter line was generated at the Monash Gene Targeting Facility through standard pronuclear injection techniques. Recombineering was used to construct the transgene from a BAC template. The mCherry-T2A-CreERT2 cassette replaces the second exon after the translation initiation site and is followed by a poly $(\mathrm{A})$ sequence. The transgene incorporates $20 \mathrm{~kb}$ upstream $\mathrm{Plzf}$, the first untranslated exon and first intron plus $1 \mathrm{~kb}$ of the second intron. Two independent founder lines were established and mice from the first line were used for the majority of experiments. $\mathrm{Z} / \mathrm{EG}$, So $\times 2^{\mathrm{GFP}}$, Oct4-GFP, $P d x 1^{\mathrm{GFP}}$ and $I d 4^{\mathrm{IRES}-\mathrm{GFP}}$ lines have been described elsewhere $25,28,32,38,40$. Hemizygous or heterozygous reporter mice were generally maintained on a mixed C57BL6/CBA background except Oct4-GFP (C57BL6/ $\mathrm{CBA} / 129 \mathrm{~T} 2 \mathrm{svJ}$ ) and Id4 $4^{\mathrm{IRES}-\mathrm{GFP}}$ (FVBN/CBA) lines. For lineage tracing, Plzf-mC/ CreER; Z/EG mice (4-6 weeks old) were injected intraperitoneally daily with $2 \mathrm{mg}$ tamoxifen (Sigma-Aldrich) in sesame oil for 5 consecutive days ${ }^{22}$. Wild-type mice for analysis of testis development and regeneration were C57BL6. Adult mice were generally harvested at 8-10 weeks of age. Busulfan (Cayman Chemical) was prepared for intraperitoneal injection as described ${ }^{22}$. Spermatogonial transplantation was performed using busulfan-conditioned C57BL6/CBA F1 recipients ${ }^{20}$. A volume of $10-15 \mu \mathrm{l}$ of donor cell suspension was microinjected via the testis efferent ducts. Recipients of cells from Oct4-GFP mice were treated with neutralising antibody to CD4 to promote immune tolerance ${ }^{35,63}$. All animal experiments were subject to approval by the Monash University Animal Ethics Committee.
}

Immunofluorescence. For frozen sections, testes were harvested and fixed in $4 \%$ paraformaldehyde (PFA) overnight at $4{ }^{\circ} \mathrm{C}$ then washed in phosphate-buffered saline (PBS) prior to cryoprotection with $30 \%$ sucrose in PBS and embedding in OCT. Sections $(8 \mu \mathrm{m})$ were processed as previously detailed ${ }^{20}$ and blocked with $10 \%$ foetal bovine serum (FBS) or donkey serum with $2 \%$ bovine serum albumin (BSA) in PBS. Whole-mount immunostaining of seminiferous tubules is described elsewhere ${ }^{48}$. For immunostaining of cultured $\mathrm{A}_{\text {undiff, cells were plated onto Lab- }}$
Tek II chamber slides (Thermo Fisher Scientific) coated with Geltrex (Thermo Fisher Scientific) and allowed to form colonies. Cells were fixed in 4\% PFA for 10 min at room temperature and washed in PBS prior to permeabilization for 10-15 min in methanol at $-20^{\circ} \mathrm{C}$. Slides were blocked and stained as for sections above. Primary antibodies were as follows: Goat anti-GFRal (AF560, 1:250), anti-c-KIT (AF1356, 1:250), anti-mouse/rat PDX1 (AF2517, 1:250), anti-PLZF (AF2944, 1:500), anti-LIN28A (AF3757, 1:500) and anti-SOX3 (AF2569, 1:250) (R\&D Systems), rabbit anti-mCherry (ab167453, 1:2000), anti-OCT4 (ab19857, 1:500), antiSALL4 (ab29112, 1:2000) (Abcam), chicken anti-GFP (ab13970, 1:5000) and rat anti-germ cell-specific antigen (TRA98, 1:500) (Abcam), rat monoclonal antimCherry clone 16D7 (Thermo Fisher Scientific, 1:2000), rabbit monoclonal antiCyclin D1 clone SP4 (1:250) and mouse monoclonal anti-DNMT3A clone 64B1446 (1:200) (Novus Biologicals), rat anti-KI67 clone SolA15 (eBioscience, 1:250), rabbit monoclonal anti-c-KIT clone D13A2 (1:400) and anti-RAR $\gamma$ clone D3A4 (1:500) (Cell Signaling Technology) and rabbit monoclonal anti-mouse EOMES clone 1219A (R\&D Systems, 1:1000). Primary antibodies were detected with appropriate Alexa Fluor-conjugated secondary antibodies (Thermo Fisher Scientific and Jackson ImmunoResearch, 1:500) and samples counterstained with DAPI prior to mounting with Vectashield mounting medium (Vector Labs). Samples were analysed on a Zeiss LSM 780 confocal microscope (Monash Micro Imaging).

Flow cytometry. To generate single-cell suspensions from adult testis, dissected seminiferous tubules were coarsely minced and washed with PBS then digested with $1 \mathrm{mg} / \mathrm{ml}$ collagenase Type IV (Sigma-Aldrich) in unsupplemented DMEM medium and $50 \mu \mathrm{g} / \mathrm{ml}$ DNase I. Tubule fragments were washed in PBS to deplete interstitial cells and peritubular myoid cells then dissociated with $0.25 \%$ trypsin in the presence of DNase I and passed through a $70 \mu \mathrm{m}$ cell strainer. For live analysis and sorting, cells were stained with FITC (1:250) or biotin-conjugated (1:500) antiCD9 clones KMC8 or MZ3 (eBioscience and Biolegend) and allophycocyanin (APC)-conjugated anti-c-KIT clone 2B8 (eBioscience, 1:500). Biotinylated antibody was detected with streptavidin conjugated to APC-eFluor 780 (eBioscience, 1:2000) and DAPI used for live/dead discrimination. Spermatogonia-enriched fractions for sorting were prepared by CD9 selection using an EasySep biotin selection kit (Stem Cell Technologies) and biotinylated CD9 antibody (Biolegend clone MZ3, 1:500) according to manufacturers instructions. For intracellular staining, testis cell suspensions were fixed in PFA then permeabilized in methanol on ice for $30 \mathrm{~min}$ or overnight at $-20{ }^{\circ} \mathrm{C}$. Fixed and permeabilized cells were washed in PBS with $2 \%$ FBS then stained with the following antibodies: chicken anti-GFP (Abcam ab13970 1:5000), eFluor 450-conjugated anti-Ki67 clone SolA15 (1:500) and phycoerythrin (PE)-conjugated anti-c-KIT clone 2B8 (1:250) (eBioscience), rabbit polyclonal antimCherry (Abcam ab167453, 1:1000), goat anti-mouse/rat PDX1 (R\&D Systems, AF2517, 1:300), rabbit anti-mouse EOMES clone 1219A (R\&D Systems, 1:1000) and Alexa 647-conjugated anti-PLZF clone 9E12 (1:2000) ${ }^{20}$. Antibodies to EOMES, GFP, mCherry and PDX1 were detected with Alexa 488 and 568 conjugated secondary antibodies (Thermo Fisher Scientific and Jackson ImmunoResearch, 1:500) Cells were analysed on an LSR Fortessa and sorted with a BD Influx Cell Sorter (BD Biosciences). Data were processed with FlowJo software.

Cell culture. $\mathrm{A}_{\text {undiff }}$ cultures were established and maintained on mitomycininactivated mouse embryonic fibroblast (MEF) feeders in StemPro-34 media (Thermo Fisher Scientific) supplemented with $10 \mathrm{ng} / \mathrm{ml}$ GDNF, $10 \mathrm{ng} / \mathrm{ml} \mathrm{bFGF}, 20$ $\mathrm{ng} / \mathrm{ml}$ EGF, $25 \mu \mathrm{g} / \mathrm{ml}$ insulin and other additives as described ${ }^{20,64}$. Cultures between passages (P) 3 and 8 were used unless indicated otherwise and harvested using Trypsin/EDTA. For analysis of cell growth and GFP expression by flow cytometry, cells were plated onto 24-well plates at indicated densities and followed over time. When analysed by flow cytometry, $\mathrm{A}_{\text {undiff }}$ were distinguished from contaminating feeders by mCherry expression. For IF, cells were plated onto fourwell chamber slides coated with Geltrex (Thermo) as detailed ${ }^{22}$. GDNF levels in conditioned media from cells cultured on 12-well plates were measured with a DuoSet ELISA system (R\&D Systems). To induce differentiation, cells were treated with $1 \mu \mathrm{m}$ retinoic acid in DMSO (Sigma-Aldrich) for $48 \mathrm{~h}$. For transplantation, cells were dissociated with Trypsin/EDTA and resuspended at $5 \times 10^{6}$ cells $/ \mathrm{ml}$ in germ cell media supplemented with $50 \mu \mathrm{g} / \mathrm{ml}$ DNase I and 10\% Trypan blue (Sigma-Aldrich). Inhibitors to MAPK (PD0325901; $5 \mu \mathrm{m})$, PI3Ka (BYL719; $1 \mu \mathrm{m}$ ) and mTOR (Torin; $0.5 \mu \mathrm{m}$ ) (Selleckchem) were dissolved in DMSO then diluted in media. Inhibitor-containing media was replaced each day for 4 days prior to cell analysis.

Microarray and $\mathbf{q R T}$-PCR. Sorted cells were lysed in TRIzol LS reagent (Thermo Fisher Scientific) then RNA was purified and DNase treated using a Direct-zol RNA Miniprep kit (Zymo Research). Gene expression in $A_{\text {undiff fractions from }}$ pooled Oct4-GFP; Plzf-mC/CreER mice (three independent sorts) was analysed with an Agilent Technologies SurePrint G3 $8 \times 60 \mathrm{~K}$ microarray at the Monash Health Translation Precinct Genomics Facility. Significance was determined by paired $t$ test $(P<0.05)$ with 1.5 -fold change cutoff. For qRT-PCR, cDNA was synthesized from isolated RNA using a Tetro cDNA synthesis kit (Bioline). Quantitative PCRs were run on a Roche LightCycler 480 using Takara Sybr Premix Ex Taq II (Clontech). Primer sequences were as previously described ${ }^{20,23}$ or 
obtained from the Harvard PCR Primer Bank (https://pga.mgh.harvard.edu/ primerbank/) and listed in Supplementary Table 1.

RNA-sequencing. RNA was purified from sorted cells and DNase treated using a Direct-zol RNA Microprep kit (Zymo Research). RNA quality and quantity was assessed using a Bioanalyser and Qubit. SPIA amplification and CDNA generation was performed using 2 ng total RNA as per Nugen Ovation RNA-seq system V2 protocol followed by Ovation Ultralow System V2 for library preparation. SPIA amplified cDNA was quantitated and $100 \mathrm{ng}$ sheared using Covaris sonication and processed. Seven cycles of amplification were used to minimize any amplification effects. Library size ( $\sim 320 \mathrm{bp})$ was checked by Bioanalyzer. Libraries were quantified by Qubit and qPCR and one equimolar pool made based upon qPCR results. Following denaturation, $200 \mathrm{pM}$ of library pool was clustered in one lane of a HiSeq 3000 8-lane flowcell using c-Bot. RNA-sequencing was performed at the Medical Genomics Facility, Monash Health Translation Precinct (MHTP). Data was processed by the Monash University Bioinformatics Platform using the RNAsik pipeline. Raw reads (FASTQ) were mapped against Ensembl reference files (FASTA and GTF) followed by read counting. Differential gene expression was performed with Degust webtool, based around the R package limma using limmavoom for statistical analysis. Differentially expressed genes were classified using the DAVID Bioinformatics Resource and KEGG pathway database ${ }^{65}$.

Single-cell PCR. Preamplified template for single cell PCR was generated with Fluidigm's C1 platform as per instrument specifications. In two independent experiments a total of 152 mCherry + CD9 + c-KIT - cells isolated from pooled Plzf-mC/CreER adult testis were captured and preamplified with 71 TaqMan probes to the following genes (those identified as differentially expressed in Oct4-

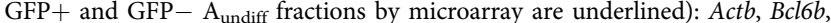
Ccnd2, Ccne1, Cd82, Cxcr4, Ddit4, Ddx4, Dmrt1, Dnmt3a, Dnmt3b, Dnmt31, Dppa2, Dppa3, Egr2, Eif4b, Elk3, Eomes, Esr1, Etv1, Etv4, Etv5, Fbxw7, Fgfr1, Fgfr2,$\overline{\text { Foxo6 }}$,

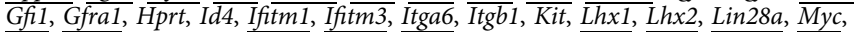
Nanos2, Nanos3, Neurod1, Ngn3, Notch1, Pax7, Pdx1, Pou $f 1$ 1, Pou $3 f_{2}$, Pousf1, Rarg, Ret, Rpl32, Rspo1, Sall1, Sall, Six2, Smad6, Snai1, Sohlh1, Sox2, Sox3, Sox10, $\overline{\text { Stra8 }}, \bar{T}, T b \times 20, \overline{T c l 1}, T$ Tet3, Thy1, Upp1, Zbtb16 and Zic2. Single-cell PCR data collection was performed with a Biomark instrument (Fluidgm). Results are expressed as $\log 2 \mathrm{Ex}=\mathrm{LOD}$ (Limit of Detection) $\mathrm{Cq}-\mathrm{Cq}$ [Gene]. The limit of detection was set to 28 . If $\log 2 \mathrm{Ex}$ value is negative, $\log 2 \mathrm{Ex}=0$. Summary statistics and plots were performed and produced using made4, caroline and gplots ${ }^{66,67}$ viSNE, a visualization tool for high-dimensional single-cell data based on the Barnes-Hut implementation of the t-Distributed Stochastic Neighbour Embedding (t-SNE) algorithm was applied to the data set ${ }^{34}$. In detail, 0.1 was added to Log2Ex values and resulting tables converted to flow cytometry standard files. The Cytobank platform (Fluidgm, South San Francisco, California) was used for generation of viSNE maps.

Single-cell RNA-sequencing. mCherry $+\mathrm{CD} 9+\mathrm{c}-\mathrm{KIT}-\mathrm{A}_{\text {undiff }}$ fractions were independently isolated from 2 Plzf-mC/CreER; Oct4-GFP adults and resuspended at $\sim 300$ viable cells $/ \mu$ in PBS containing $0.04 \%$ BSA. Library construction was performed using a $10 \times$ Chromium controller with the Chromium Single Cell $3^{\prime}$ Reagent Kit V2. Both libraries were sequenced in one high-output lane of an Illumina NextSeq in single-read 150b format (115b effective read length). Singlecell (sc) RNA-seq processing and analysis was performed with Cell Ranger software (10× Genomics, Inc.) and Monocle ${ }^{36,68,69}$. scRNA-seq samples were demultiplexed with cellranger mkfastq. Sequencing reads were aligned to a custom mouse genome (Cell Ranger mm10 version 1.2.0, including GFP and mCherry sequences as extra chromosomes). The annotation file was modified to count reads assigned to both GFP and mCherry cassettes and UMIs counted with cellranger count. Both libraries were aggregated and normalized by depth, to have the same of number of mapped reads, with cellranger aggr. Gene expression matrix, containing both cell and expression details, were processed for downstream analysis using both cellRanger's load_cellranger_matrix and Monocle's newCellDataSet function (lowerDetectioLimit $=0.5$ and expressionFamily $=$ negbinomial.size () ).

Analysis was performed using components of Monocle's package unless otherwise stated. Cell counts were normalised with the estimateSizeFactors function previously discarding mitochondrial and ribosomal associated genes ${ }^{70}$. Cell gene expression was detected using detectGenes $\left(\min \_\right.$expr $\left.=0.1\right)$ and genes not expressed in at least 175 cells were discarded. Dispersion was estimated for the remaining gene set using estimateDispersions ${ }^{70}$. Cells with a total number of mRNA $(\log 10)$ within two standard deviations from the mean were kept for further analysis.

In a first instance, cell classification was performed with classifyCells based on the following criteria: contaminant cells were defined as those cells that expressed at least one transcript of Acta2, Icam 2 or Cdh 5 marker genes; germ cells were those cells that expressed at least one transcript of Zbtb16, Ddx4 or Sall4 and did not express any of the contaminant cell marker genes. Only those cells classified as germ cell were used for downstream analyses. Cells with no detectable expression of the housekeeping gene Gapdh (senescent) were also excluded from the analysis.

For single-cell trajectory analysis, germ cells were ordered in a semi-supervised mode using a cell type classification based on the following criteria: transitional cells were defined as those cells that express at least one transcript of both stem (Etv5 and Gfra1) and progenitor (Upp1 and Sox3) marker genes; stem cells were defined as those cells that expressed at least one transcript of the stem cell marker genes but did not express progenitor marker genes; progenitor cells were defined as cells that expressed at least one transcript of the defined progenitor marker genes and did not express stem cell marker genes. A minor fraction of cells was not classified as either stem or progenitor cells based on the described markers and were referred to as undefined undifferentiated. The makerDiffTable function (residualModelFormulaStr $=$ " $\sim$ Library+ num_genes_expressed") was used to identify those genes that were co-expressed with markers that define cell type hierarchy described above (top 2500 genes based on q value were selected as ordering genes). Dimensionality reduction was performed with reduceDimension using the discriminative dimensionality reduction with trees (DDRTree) method and $\log$ normalization. Cells were ordered along the trajectory with orderCells $($ reverse $=\mathrm{T})$. Differential gene expression test along the cell trajectory was performed with differentialGeneTest (fullModelFormulaStr $=$ " $\sim$ sm.ns (Pseudotime) + Library+ num_genes_expressed" and reducedModelFormulaStr $=$ " Library+ num_genes_expressed"). Cell trajectory, gene expression across pseudotime and heatmap of pseudotime plots were created with plot_cell_trajectory, plot_genes_in_pseudotime $\left(\min \_\right.$expr $=0.1$, trend_formula $=" \sim$ sm.ns(Pseudotime, $\mathrm{df}=4) "$ ) and plot_pseuodtime_heatmap respectively.

Statistical analysis. Assessment of statistical significance was performed using a two-tailed unpaired $t$-test (GraphPad Prism). $P$-values are indicated as follows: ${ }^{*} P$ $<0.05 ;{ }^{* *} P<0.01$; ${ }^{* *} P<0.001 ;{ }^{* * *} P<0.0001$; not significant (ns) $P>0.05$. For mouse experiments, no statistical method was used to predetermine sample sizes and no specific randomization or blinding methods were used.

Data availability. Microarray and RNA-Seq data are deposited in the Gene Expression Omnibus (GEO) database as a SuperSeries with accession number GSE107256. All other relevant data are available from the corresponding author on request.

Received: 28 March 2018 Accepted: 22 May 2018 Published online: 19 July 2018

\section{References}

1. Donati, G. \& Watt, F. M. Stem cell heterogeneity and plasticity in epithelia. Cell Stem Cell 16, 465-476 (2015).

2. de Rooij, D. G. Stem cells in the testis. Int. J. Exp. Pathol. 79, 67-80 (1998).

3. Shirakawa, T. et al. An epigenetic switch is crucial for spermatogonia to exi the undifferentiated state toward a Kit-positive identity. Development 140, 3565-3576 (2013).

4. Nakagawa, T., Sharma, M., Nabeshima, Y., Braun, R. E. \& Yoshida, S. Functional hierarchy and reversibility within the murine spermatogenic stem cell compartment. Science 328, 62-67 (2010).

5. Chan, F. et al. Functional and molecular features of the Id4+germline stem cell population in mouse testes. Genes Dev. 28, 1351-1362 (2014).

6. Kanatsu-Shinohara, M. \& Shinohara, T. Spermatogonial stem cell self-renewal and development. Annu. Rev. Cell. Dev. Biol. 29, 163-187 (2013).

7. Nagano, M. C. Homing efficiency and proliferation kinetics of male germ line stem cells following transplantation in mice. Biol. Reprod. 69, 701-707 (2003).

8. Helsel, A. R. et al. ID4 levels dictate the stem cell state in mouse spermatogonia. Development 144, 624-634 (2017).

9. Nakagawa, T., Nabeshima, Y. \& Yoshida, S. Functional identification of the actual and potential stem cell compartments in mouse spermatogenesis. Dev. Cell 12, 195-206 (2007)

10. Hara, K. et al. Mouse spermatogenic stem cells continually interconvert between equipotent singly isolated and syncytial states. Cell Stem Cell 14, 658-672 (2014).

11. Ikami, K. et al. Hierarchical differentiation competence in response to retinoic acid ensures stem cell maintenance during mouse spermatogenesis. Development 142, 1582-1592 (2015).

12. Suzuki, H. et al. SOHLH1 and SOHLH2 coordinate spermatogonial differentiation. Dev. Biol. 361, 301-312 (2012).

13. Carrieri, C. et al. A transit-amplifying population underpins the efficient regenerative capacity of the testis. J. Exp. Med. 214, 1631-1641 (2017).

14. Meng, X. et al. Regulation of cell fate decision of undifferentiated spermatogonia by GDNF. Science 287, 1489-1493 (2000).

15. Ishii, K., Kanatsu-Shinohara, M., Toyokuni, S. \& Shinohara, T. FGF2 mediates mouse spermatogonial stem cell self-renewal via upregulation of Etv5 and Bcl6b through MAP2K1 activation. Development 139, 1734-1743 (2012).

16. Oatley, J. M., Avarbock, M. R., Telaranta, A. I., Fearon, D. T. \& Brinster, R. L. Identifying genes important for spermatogonial stem cell self-renewal and survival. Proc. Natl Acad. Sci. USA 103, 9524-9529 (2006). 
17. Lee, J. et al. Genetic reconstruction of mouse spermatogonial stem cell selfrenewal in vitro by Ras-cyclin D2 activation. Cell Stem Cell 5, 76-86 (2009).

18. Oatley, J. M., Avarbock, M. R. \& Brinster, R. L. Glial cell line-derived neurotrophic factor regulation of genes essential for self-renewal of mouse spermatogonial stem cells is dependent on Src family kinase signaling. J. Biol. Chem. 282, 25842-25851 (2007).

19. Costoya, J. A. et al. Essential role of Plzf in maintenance of spermatogonial stem cells. Nat. Genet. 36, 653-659 (2004).

20. Hobbs, R. M., Seandel, M., Falciatori, I., Rafii, S. \& Pandolfi, P. P. Plzf regulates germline progenitor self-renewal by opposing mTORC1. Cell 142, 468-479 (2010).

21. Buaas, F. W. et al. Plzf is required in adult male germ cells for stem cell selfrenewal. Nat. Genet. 36, 647-652 (2004).

22. Chan, A. L. et al. Germline stem cell activity is sustained by SALL4-dependent silencing of distinct tumor suppressor genes. Stem Cell Rep. 9, 956-971 (2017).

23. Hobbs, R. M. et al. Functional antagonism between Sall4 and Plzf defines germline progenitors. Cell Stem Cell 10, 284-298 (2012).

24. Dann, C. T. et al. Spermatogonial stem cell self-renewal requires OCT4, a factor downregulated during retinoic acid-induced differentiation. Stem Cells 26, 2928-2937 (2008).

25. Novak, A., Guo, C., Yang, W., Nagy, A. \& Lobe, C. G. Z/EG, a double reporter mouse line that expresses enhanced green fluorescent protein upon Cre-mediated excision. Genesis 28, 147-155 (2000).

26. Kanatsu-Shinohara, M., Toyokuni, S. \& Shinohara, T. CD9 is a surface marker on mouse and rat male germline stem cells. Biol. Reprod. 70, 70-75 (2004)

27. Oulad-Abdelghani, M. et al. Characterization of a premeiotic germ cellspecific cytoplasmic protein encoded by Stra8, a novel retinoic acid-responsive gene. J. Cell. Biol. 135, 469-477 (1996).

28. Arnold, K. et al. Sox2(+) adult stem and progenitor cells are important for tissue regeneration and survival of mice. Cell Stem Cell 9, 317-329 (2011).

29. Ko, K. et al. Induction of pluripotency in adult unipotent germline stem cells. Cell Stem Cell 5, 87-96 (2009).

30. Ohbo, K. et al. Identification and characterization of stem cells in prepubertal spermatogenesis in mice. Dev. Biol. 258, 209-225 (2003).

31. Ohmura, M. et al. Spatial analysis of germ stem cell development in Oct-4/EGFP transgenic mice. Arch. Histol. Cytol. 67, 285-296 (2004)

32. Szabo, P. E., Hubner, K., Scholer, H. \& Mann, J. R. Allele-specific expression of imprinted genes in mouse migratory primordial germ cells. Mech. Dev. 115, $157-160$ (2002).

33. Wu, X., Goodyear, S. M., Tobias, J. W., Avarbock, M. R. \& Brinster, R. L. Spermatogonial stem cell self-renewal requires ETV5-mediated downstream activation of Brachyury in mice. Biol. Reprod. 85, 1114-1123 (2011).

34. Amir el, A. D. et al. viSNE enables visualization of high dimensional single-cell data and reveals phenotypic heterogeneity of leukemia. Nat. Biotechnol. 31, 545-552 (2013).

35. Aloisio, G. M. et al. PAX7 expression defines germline stem cells in the adult testis. J. Clin. Invest. 124, 3929-3944 (2014).

36. Trapnell, C. et al. The dynamics and regulators of cell fate decisions are revealed by pseudotemporal ordering of single cells. Nat. Biotechnol. 32, 381-386 (2014).

37. Bernardo, A. S., Hay, C. W. \& Docherty, K. Pancreatic transcription factors and their role in the birth, life and survival of the pancreatic beta cell. Mol. Cell. Endocrinol. 294, 1-9 (2008).

38. Holland, A. M., Micallef, S. J., Li, X., Elefanty, A. G. \& Stanley, E. G. A mouse carrying the green fluorescent protein gene targeted to the Pdx1 locus facilitates the study of pancreas development and function. Genesis $\mathbf{4 4}$, 304-307 (2006).

39. Yoshida, S., Sukeno, M. \& Nabeshima, Y. A vasculature-associated niche for undifferentiated spermatogonia in the mouse testis. Science 317, 1722-1726 (2007).

40. Best, S. A. et al. Dual roles for Id4 in the regulation of estrogen signaling in the mammary gland and ovary. Development 141, 3159-3164 (2014).

41. Tokue, M. et al. SHISA6 confers resistance to differentiation-promoting Wnt/ beta-catenin signaling in mouse spermatogenic stem cells. Stem Cell Rep. 8, 561-575 (2017).

42. Signer, R. A., Magee, J. A., Salic, A. \& Morrison, S. J. Haematopoietic stem cells require a highly regulated protein synthesis rate. Nature 509, 49-54 (2014).

43. Hasegawa, K., Namekawa, S. H. \& Saga, Y. MEK/ERK signaling directly and indirectly contributes to the cyclical self-renewal of spermatogonial stem cells. Stem Cells 31, 2517-2527 (2013)

44. Jonsson, J., Ahlgren, U., Edlund, T. \& Edlund, H. IPF1, a homeodomain protein with a dual function in pancreas development. Int. J. Dev. Biol. 39, 789-798 (1995).

45. Brissova, M. et al. Reduction in pancreatic transcription factor PDX-1 impairs glucose-stimulated insulin secretion. J. Biol. Chem. 277, 11225-11232 (2002).

46. Takashima, S. et al. Functional differences between GDNF-dependent and FGF2-dependent mouse spermatogonial stem cell self-renewal. Stem Cell Rep. 4, 489-502 (2015).
47. Goertz, M. J., Wu, Z., Gallardo, T. D., Hamra, F. K. \& Castrillon, D. H. Foxol is required in mouse spermatogonial stem cells for their maintenance and the initiation of spermatogenesis. J. Clin. Invest. 121, 3456-3466 (2011).

48. Hobbs, R. M. et al. Distinct germline progenitor subsets defined through Tsc2-mTORC1 signaling. EMBO Rep. 16, 467-480 (2015).

49. McLean, D. J., Friel, P. J., Johnston, D. S. \& Griswold, M. D. Characterization of spermatogonial stem cell maturation and differentiation in neonatal mice. Biol. Reprod. 69, 2085-2091 (2003).

50. Shinohara, T., Orwig, K. E., Avarbock, M. R. \& Brinster, R. L. Remodeling of the postnatal mouse testis is accompanied by dramatic changes in stem cell number and niche accessibility. Proc. Natl Acad. Sci. USA 98, 6186-6191 (2001).

51. Ebata, K. T., Zhang, X. \& Nagano, M. C. Male germ line stem cells have an altered potential to proliferate and differentiate during postnatal development in mice. Biol. Reprod. 76, 841-847 (2007).

52. van Keulen, C. J. \& de Rooij, D. G. Spermatogenetic clones developing from repopulating stem cells surviving a high dose of an alkylating agent. Cell Tissue Kinet. 8, 543-551 (1975).

53. Lord, T. \& Oatley, J. M. A revised Asingle model to explain stem cell dynamics in the mouse male germline. Reproduction 154, R55-R64 (2017).

54. Pech, M. F. et al. High telomerase is a hallmark of undifferentiated spermatogonia and is required for maintenance of male germline stem cells. Genes Dev. 29, 2420-2434 (2015).

55. Wuidart, A. et al. Quantitative lineage tracing strategies to resolve multipotency in tissue-specific stem cells. Genes Dev. 30, 1261-1277 (2016).

56. Babovic, S. \& Eaves, C. J. Hierarchical organization of fetal and adult hematopoietic stem cells. Exp. Cell Res. 329, 185-191 (2014).

57. Bowie, M. B. et al. Identification of a new intrinsically timed developmental checkpoint that reprograms key hematopoietic stem cell properties. Proc. Natl Acad. Sci. USA 104, 5878-5882 (2007).

58. Copley, M. R. et al. The Lin28b-let-7-Hmga2 axis determines the higher selfrenewal potential of fetal haematopoietic stem cells. Nat. Cell Biol. 15, 916-925 (2013)

59. Kim, I., Saunders, T. L. \& Morrison, S. J. Sox17 dependence distinguishes the transcriptional regulation of fetal from adult hematopoietic stem cells. Cell 130, 470-483 (2007).

60. Sharpe, R. M., McKinnell, C., Kivlin, C. \& Fisher, J. S. Proliferation and functional maturation of Sertoli cells, and their relevance to disorders of testis function in adulthood. Reproduction 125, 769-784 (2003).

61. Zohni, K., Zhang, X., Tan, S. L., Chan, P. \& Nagano, M. C. The efficiency of male fertility restoration is dependent on the recovery kinetics of spermatogonial stem cells after cytotoxic treatment with busulfan in mice. Hum. Reprod. 27, 44-53 (2012).

62. Mullaney, B. P. \& Skinner, M. K. Basic fibroblast growth factor (bFGF) gene expression and protein production during pubertal development of the seminiferous tubule: follicle-stimulating hormone-induced Sertoli cell bFGF expression. Endocrinology 131, 2928-2934 (1992).

63. Kanatsu-Shinohara, M. et al. Long-term proliferation in culture and germline transmission of mouse male germline stem cells. Biol. Reprod. 69, 612-616 (2003).

64. Martin, L. A. \& Seandel, M. Serial enrichment of spermatogonial stem and progenitor cells (SSCs) in culture for derivation of long-term adult mouse SSC lines. J. Vis. Exp. 72, e50017 (2013).

65. Huang da, W., Sherman, B. T. \& Lempicki, R. A. Systematic and integrative analysis of large gene lists using DAVID bioinformatics resources. Nat. Protoc. 4, 44-57 (2009).

66. Culhane, A. C., Thioulouse, J., Perriere, G. \& Higgins, D. G. MADE4: an R package for multivariate analysis of gene expression data. Bioinformatics 21, 2789-2790 (2005)

67. Schruth, D. Caroline: a collection of database, data structure, visualization, and utility functions for R. Preprint at http://CRAN.R-project.org/ package $=$ caroline (2013).

68. Qiu, X. et al. Reversed graph embedding resolves complex single-cell trajectories. Nat. Methods 14, 979-982 (2017).

69. Zheng, G. X. et al. Massively parallel digital transcriptional profiling of single cells. Nat. Commun. 8, 14049 (2017).

70. Love, M. I., Huber, W. \& Anders, S. Moderated estimation of fold change and dispersion for RNA-seq data with DESeq2. Genome Biol. 15, 550 (2014).

\section{Acknowledgements}

We thank Jeanette Rientjes and Jose Gonzalez of the Monash Gene Targeting Facility for generating the Plzf-mC/CreER transgenic line and technicians of the Monash Animal Research Platform for animal care. We would also like to thank Patrick Western, Anand Hardikar, Jane Visvader and Alex Swarbrick for supplying reporter mice. We are very grateful to Antonella Papa for helpful discussions and comments plus Marco Seandel for advice on spermatogonial transplantation. We acknowledge the facilities, scientific and technical assistance of Monash Micro Imaging, FlowCore and Micromon of Monash University. This work was supported by an Australian Research Council (ARC) Future 
Fellowship to R.M.H. and by the Stem Cells Australia ARC Special Research Initiative. J. M. was supported by a fellowship from the Sigrid Jusélius Foundation. J.M.P. is a Viertel Senior Medical Research Fellow. The Australian Regenerative Medicine Institute is supported by grants from the State Government of Victoria and the Australian Government.

\section{Author contributions}

R.M.H., J.M., H.M.L. and J.M.P. conceived and designed the study. H.M.L., J.M., A.C., J. M.D.L., M.S. and R.M.H. performed experiments. H.M.L., J.M., F.J.R., C.M.N., J.M.P. and R.M.H. analysed the data. R.M.H. and J.M.P. wrote the paper.

\section{Additional information}

Supplementary Information accompanies this paper at https://doi.org/10.1038/s41467018-04827-z.

Competing interests: The authors declare no competing interests.

Reprints and permission information is available online at http://npg.nature.com/ reprintsandpermissions/
Publisher's note: Springer Nature remains neutral with regard to jurisdictional claims in published maps and institutional affiliations.

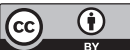

Open Access This article is licensed under a Creative Commons Attribution 4.0 International License, which permits use, sharing, adaptation, distribution and reproduction in any medium or format, as long as you give appropriate credit to the original author(s) and the source, provide a link to the Creative Commons license, and indicate if changes were made. The images or other third party material in this article are included in the article's Creative Commons license, unless indicated otherwise in a credit line to the material. If material is not included in the article's Creative Commons license and your intended use is not permitted by statutory regulation or exceeds the permitted use, you will need to obtain permission directly from the copyright holder. To view a copy of this license, visit http://creativecommons.org/ licenses/by/4.0/.

(C) The Author(s) 2018 